\title{
The public health importance of Ascaris lumbricoides
}

\author{
P. O’LORCAIN and C. V. HOLLAND* \\ Department of Zoology, Trinity College, Dublin 2, Ireland
}

\begin{abstract}
S U M MARY
Numerous studies have shown that anthelminthic treatment can be effective in improving growth rates when given to malnourished children with ascariasis. Recent investigations have also indicated that Ascaris infections can affect mental processing in some school children. Poor socio-economic conditions are among the key factors linked with higher prevalences of ascariasis, as are defaecation practices, geophagia, cultural differences relating to personal and food hygiene, occupational necessity, agricultural factors, housing style, social class and gender. Chemotherapy is currently the major tool used for the strategic control of ascariasis as a short-term goal. In the long term, improvements in hygiene and sanitation are thought to aid long-term control considerably. Targeted treatment, especially when aimed at schoolchildren, has been a major focus of recent control efforts in some areas. Universal treatment reaches more people and thus decreases further aggregate morbidity, especially in nutritionally vulnerable preschool-age children. Selective treatment requires technical effort to identify heavily infected individuals; acceptance by the community may vary in less educated populations when some individuals receive treatment and others do not. Child-targeted treatment may be more cost-effective than population treatment in reducing the number of disease cases and, in high transmission areas, expanding coverage of a population can be a more cost-effective strategy than increasing the frequency of treatment.
\end{abstract}

Key words: Ascaris lumbricoides, child growth, cognitive performance, chemotherapy control strategies, cost effectiveness.

INTRODUCTION

PUBLIC HEALTH RELEVANCE AND RECENT

GLOBAL ESTIMATES

Ascaris lumbricoides is a remarkably infectious and persistent parasite that infects a quarter of the world's population (Pawlowski \& Arfaa, 1984; Crompton, 1994). It has been widely recognized that ascariasis plays a major role in the aetiology of childhood malnutrition (Crompton, 1992). Global numbers of infections have been estimated to be 800-1000 millions (Walsh \& Warren, 1979), and more recently about 1400-1500 millions (Chan et al. 1994; WHO, 1996a). De Silva, Chan \& Bundy (1997a) calculated, using sensitivity analysis of theoretical models of parasite distributions, approximately 1300 million infections globally. In order to calculate updated estimates of possible associated morbidity, epidemiological studies have developed methods for estimating the relationship between prevalence and mean intensity and potential morbidity which incorporate age classes and geographical heterogeneity (Chan et al. 1994). These estimates are based on empirical data and chosen to be relatively conservative. According to these estimates, the morbidity associated with $A$. lumbricoides infection amounts to approximately 120-220 million cases, $8-15 \%$ of the total number infected (Albonico, Crompton \& Savioli, 1999).

* Corresponding author. Tel: +353-1-6081096, Fax: +353-1-6778094. E-mail: cholland@tcd.ie

\section{E P IDEMIOLOGY}

\section{Geographic distribution, prevalence and intensity}

An estimated $73 \%$ of $A$. lumbricoides infections are present in Asia, while about $12 \%$ are located in Africa and $8 \%$ in Latin America (Peters, 1978). However, human ascariasis is cosmopolitan, with infection occurring in both temperate and tropical environments. In Africa, for example, the prevalence is low where the climate is arid but high where conditions are wet and warm (Crompton \& Tulley, 1987; Prost, 1987) as these are ideal for egg survival and embryonation.

Poor socio-economic conditions are linked with higher prevalences of ascariasis. About one third of the population in the cities of some developing countries live in slums and shanty towns where the prevalence and intensity of $A$. lumbricoides is significantly increasing due to favourable conditions of transmission (Crompton \& Savioli, 1993). Generally, the poorer the quality of housing and community services, the more likely $A$. lumbricoides will persist and flourish (Holland et al. 1988). Ascaris infections may cluster in certain households, with heavier infections being recorded from households with more family members (Forrester et al. 1988; Asaolu et al. 1992). Kightlinger, Seed \& Kightlinger (1998) found that aggregations of $A$. lumbricoides in children in Madagascar were associated with housing style, ethnicity and agricultural factors. Other factors contributing to infection include defaecation practices (Haswell-Elkins, Elkins \& Anderson, 1989) and 
geophagia (Wong, Bundy \& Golden 1988) but also cultural differences relating to personal and food hygiene (Haswell-Elkins et al. 1989; Kan, Guyatt \& Bundy 1989), occupational necessity (Chandiwana, Bradley \& Chombo, 1989), social class (Machado et al. 1996) and gender (McCullough, 1974; Cross et al. 1975; Arfaa \& Ghadirian, 1977; Shield, Scrimgeour \& Vaterlaws, 1980; Harinasuta \& Charoenlarp, 1980; Cabrera \& Valeza, 1980; Kightlinger et al. 1998; Hall et al. 1999).

Prevalence is defined as the number of egg-positive cases in a population whereas intensity is the mean number of worms per person (Crompton, 1994). From an epidemiological perspective, prevalence can be regarded as a rather inaccurate measure of a community's ascariasis burden compared to that of intensity. Prevalence values for geographical regions or countries are still useful however, for defining the public health significance of a problem, for determining health priorities and highlighting the need for control (Crompton, 1994; Walsh \& Warren, 1979), and also for estimating the number of doses of anthelminthic drugs required.

Knowledge of the intensity of $A$. lumbricoides infection is important not only in terms of morbidity (Pawlowski \& Davis, 1989) but also in terms of the dynamics of infection, particularly on the presence of heavily infected individuals and the consequences of disease (Anderson \& May, 1991). A key feature of the biology of helminth infections is that the occurrence of disease is related to the intensity of infection. A few worms tend to be asymptomatic but as more worms are acquired, signs and symptoms of disease are more likely to occur (Bundy et al. 1990). Intensity is also important with regard to the effective implementation and monitoring of control programmes (WHO, 1987). Practical considerations determine that, in general, faecal egg counts have to be used as measures of intensity despite their variability, rather than direct counts of total worms harboured per host. The occurrence of disease is not just related to the number of worms in the host, but is likely also to be related to duration of infection and to the host's background health status (Albonico et al. 1999).

\section{Diagnosis of infection}

Intensity is best, but least frequently, measured directly by counting the number of worms passed in the stools after anthelminthic treatment (Croll \& Ghadirian, 1981). The presence of Ascaris eggs in stool samples in contrast is determined with a direct smear technique or quantified using the Kato Katz or other quantitative methods (Thienpont, Rochette \& Vanparijs, 1986; WHO, 1992). Egg counts give an indirect measure of the intensity of infection and are expressed as epg (eggs per gram of faeces). It is assumed that a greater faecal egg count usually indicates the presence of a greater number of sexually mature female $A$. lumbricoides worms in the infected individual (Crompton, 1994). However, difficulties may arise when interpreting faecal egg counts since density-dependent constraints on fecundity may disguise the true number of worms present (Hall, 1982; Keymer, 1982; Thein Hlaing et al. 1984; Holland et al. 1989; Forrester \& Scott, 1990). In general, however, egg counts are usually consistent with worm burden (Forrester \& Scott, 1990). Because the Kato Katz method is relatively sensitive, quick, inexpensive and simple to perform, stool sampling and examination offers the best method for the standard investigation of the epidemiology of $A$. lumbricoides in humans (Crompton, 1994; WHO, $1985 a)$.

\section{Morphology and life history}

The morphological characteristics of $A$. lumbricoides are summarized in Table 1. Ascaris is the largest of the common nematode parasites of man and has a relatively simple life cycle (Fig. 1). One female worm has the potential to produce over 200000 eggs per day (Sinniah, 1982). Eggs are passed in the faeces in the unembryonated state (Stephenson \& Holland, 1987). It is estimated that $10^{14}$ eggs pass daily into the global environment (WHO, 1981). Egg survival, once infective, is variable up to a period of 15 years (WHO, 1967; Krasnonos, 1978; Storey \& Phillips, 1985) but most are thought to be destroyed soon after passage, although many will embryonate to produce second stage larvae if provided with adequate moisture, oxygen and shade (Crompton, 1994).

Humans contract ascariasis by ingestion of embryonated eggs through faecal contamination. Because the eggs are invariably sticky, they may be found adhering to utensils, furniture, money, fruit, vegetables, door handles and fingers in endemic areas (Kagei, 1983). In a study of 51 Jamaican children (aged 7-12 years) living in 2 children's homes, Wong et al. (1991) estimated that the mean rate of ingestion of Ascaris eggs was 9-20 per year. A comparison between estimated exposure and observed worm burdens suggested that between $12 \%$ and $90 \%$ of eggs ingested developed into adult worms. As infective eggs can occur in the air and household dust, there is risk of exposure by simply inhaling and swallowing in hyperendemic areas (WHO, 1967; Bidinger, Crompton \& Arnold, 1981; Kroeger et al. 1992).

When eggs hatch in the duodenum, the larvae migrate through the liver, lungs and upper alimentary tract. When they reach the small intestine they mature into adult worms (Thein Hlaing, 1993). Many of the larvae are destroyed on their journey through the host as they are lost in inappropriate tissues (Stephenson \& Holland, 1987). The cycle 
Table 1. Morphological and life history characteristics of Ascaris lumbricoides

\begin{tabular}{ll}
\hline \hline Characteristic & Observation \\
\hline Adult lifespan & $1-2$ years \\
Adult worm size range & $150-300+\mathrm{mm}$ long; $-4 \mathrm{~mm}$ wide \\
$\quad$ Male & $200-350+\mathrm{mm}$ long; $3-6 \mathrm{~mm}$ wide \\
Female & $60-70 \mu \mathrm{m}$ long; $40-50 \mu \mathrm{m}$ wide \\
Ascaris egg dimensions & $10-14$ days at $30 \pm 2{ }^{\circ} \mathrm{C} ; 45-55$ days \\
Embryonation duration & at $17 \pm 1{ }^{\circ} \mathrm{C}$ \\
& $134,462-358,759$ eggs $/$ female $/$ day \\
Fecundity & $2-8$ days post infection \\
Tissue migration & $7-14$ days post infection \\
$\quad$ Small intestine to liver & $14-20$ days post infection \\
Liver to lungs & jejunum \\
Lungs to small intestine & $67-76$ days; 67 days in children $<4$ years \\
Normal location of adult worms & \\
Prepatent period &
\end{tabular}

Based on information from Beaver et al. (1984); Sinniah (1982); Stephenson \& Holland (1987); Pawlowski \& Arfaa (1984); Yoshida (1919); Nichols (1956); Takata (1951); Akamatsu (1959); Anderson (1982).

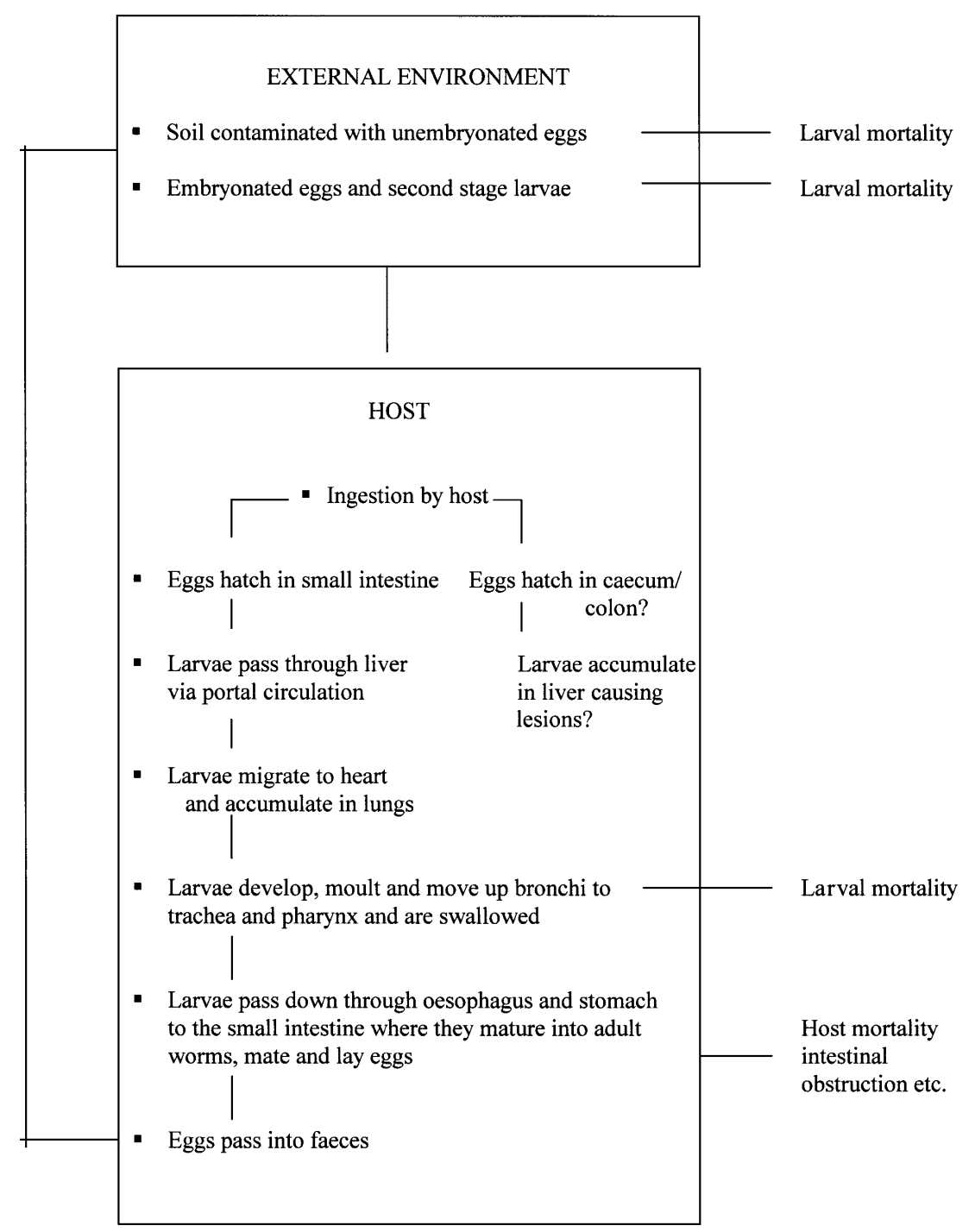

Fig. 1. Life history of Ascaris lumbricoides based on Crompton (1994) and Murrell et al. (1997). 
from ingestion of eggs to the production of fertilised eggs by mature $A$. lumbricoides takes 2-3 months (Vogel \& Minning, 1942) (Fig. 1). Adult worms can survive for 1-2 years and female worms can generate eggs for a period of one year although some may continue as long as 20 months (Hobo, 1956).

The widely held concept of the migratory pattern of A. lumbricoides in humans derives primarily from experiments employing abnormal hosts such as guinea pigs, mice and rats as hosts (Grove, 1990). However, Murrell et al. (1997) have demonstrated that $A$. suum $\mathrm{L}_{2}$ larvae in the pig host penetrate the mucosa of the caecum and colon en route to the liver, rather than doing so in the jejunum of the small intestine as is widely described. This finding raises important questions as to whether these two species share similar migratory and development patterns in their hosts, and may suggest that the potential for liver damage in humans is greater than is currently regarded.

Uncertainty exists as to whether or not $A$. lumbricoides from humans and $A$. suum from pigs are the same species, and the possibility of cross infection with $A$. suum cannot be ruled out (Lysek, 1967; Galvin, 1968; Beaver, Jung \& Cupp, 1984; Anderson, 1995), particularly in endemic regions where pigs and humans live in close proximity to one another, or where the excreta of both humans and pigs are used as a fertiliser (Peng et al. 1996). Evidence from Central and North America indicates that, genetically, the two parasite populations appear to be reproductively isolated (Anderson, RomeroAbal \& Jaenike, 1993). In contrast, Peng et al. (1998) found no significant heterogeneity in the genetic composition of the Ascaris infra-populations in both humans and pigs, perhaps because of agricultural practices in China, which include the use of nightsoil (human faeces and urine) as fertiliser on food crops that have resulted in a random distribution of alleles within the parasite populations over time.

\section{Population biology}

Intensity peaks in the 5 to 15 -year-olds and declines markedly in adults. Adults continue to be infected as they age but their worm burdens are significantly lower than those of children. Explanations for this difference likely hinge on a combination of socioenvironmental and immunological factors (Crompton, 1994). Exposure to repeated infection with $A$. lumbricoides during early life may induce some level of protective immunity. Re-infection studies show that when people have been kept free of infection through regular use of anthelminthic drugs, the prevalence of the infections may increase above the pre-treatment value after treatment ceases, perhaps because resistance to the infection has been weakened or because of lack of stimulus from resident worms (Crompton, 1994).
In addition, individuals can show statistically significant correlations in numbers of worms harboured after several rounds of treatment (Holland et al. 1989). People (especially children) with heavy or light worm loads, either as a group or as individuals, tend to re-acquire, respectively, heavy or light intensities of infections in terms of epg or worm number per individual (Thein Hlaing et al. 1987). This phenomenon, termed predisposition, is widely recognized; however, not all individuals will return to the same infection intensity after treatment. Evidence as to the underlying mechanisms responsible remains elusive. The numbers of Ascaris worms within a host population are not normally distributed but follow an aggregated or overdispersed frequency distribution (Holland et al. 1989). This means that most hosts will harbour few or no worms while a small proportion of hosts will carry heavy worm burdens. Heavily infected individuals are more at risk from morbidity and mortality and also act as significant contributors of potentially infective stages in the environment.

Only a few studies have attempted to explain the mechanisms behind the observed predisposition to Ascaris in humans. Holland et al. (1992) found, in a study of class I HLA antigen distribution among children predisposed to heavy, light or no infection with Ascaris, that individuals who remained consistently uninfected despite exposure to infection lacked the A30/31 antigen. In another study of the same group of 5-15 year old Nigerians, immunity to Ascaris was associated with higher levels of serum ferritin, C-reactive protein and eosinophil cationic protein, indicating an ongoing acute phase of infection or some inflammatory process (McSharry et al. 1999). In contrast, children who were predisposed to the infection had little serological evidence of inflammation despite their high parasite burdens. In addition, $\mathrm{IgE}$ antibody responses in conjunction with inflammatory processes appeared to be associated with natural immunity to ascariasis. In an important recent study, Williams-Blangero et al. (1999) provided evidence for a strong genetic component accounting for between $30 \%$ and $50 \%$ of the variation in Ascaris worm burden among over 1200 individuals from a single pedigree in the Jirel population of East Nepal.

\section{CLINICAL FEATURES OF ASCARIASIS}

The symptoms associated with migration of $A$. lumbricoides larvae through the liver and lungs have rarely been systematically studied at community level (Table 2) (Stephenson \& Holland, 1987). Furthermore, studies involving the treatment of larval infections are not yet possible as there is no conclusive evidence that any anthelminthic used against intestinal ascariasis is also effective against 
Table 2. Clinical features and potential nutritional outcomes associated with Ascaris lumbricoides infection

\begin{tabular}{|c|c|c|c|}
\hline Stage & Event & Clinical features & Potential Nutritional Outcome \\
\hline Larval migration & $\begin{array}{l}\text { Migration of } \\
\text { larvae through } \\
\text { lungs }\end{array}$ & $\begin{array}{l}\text { Pneumonitis, including: } \\
\text { asthma } \\
\text { cough } \\
\text { dyspnea } \\
\text { substernal pain } \\
\text { Conjunctivitis } \\
\text { Convulsions } \\
\text { Eosinophilia } \\
\text { Fever } \\
\text { Skin rash }\end{array}$ & $\begin{array}{l}\text { ? Decreased food intake } \\
- \\
\text { Decreased food intake } \\
- \\
\text { Increased nitrogen loss } \\
\text { ? Decreased food intake }\end{array}$ \\
\hline Migration/oviposition & $\begin{array}{l}\text { Presence of } \\
\text { juveniles and } \\
\text { patent adult } \\
\text { worms in } \\
\text { small intestine }\end{array}$ & $\begin{array}{l}\text { Abdominal distension } \\
\text { Abdominal pain } \\
\text { Colic } \\
\text { Nausea } \\
\text { Vomiting } \\
\text { Anal itching } \\
\text { Anorexia } \\
\text { Disordered small bowel pattern } \\
\text { D-xylose and lactose malabsorption } \\
\text { Enterocolitis } \\
\text { Fat malabsorption } \\
\text { Intermittent diarrhoea } \\
\text { Jejunal mucosal abnormalities } \\
\text { Protein malabsorption } \\
\text { Restlessness } \\
\text { Vitamin A malabsorption }\end{array}$ & $\begin{array}{l}\text { Decreased food intake } \\
\text { Decreased food intake } \\
\text { Decreased food intake } \\
\text { Decreased food intake } \\
\text { Increased nutrient loss } \\
- \\
\text { Decreased food intake } \\
\text { ? Malabsorption } \\
\text { Increased carbohydrate } \\
\quad \text { excretion } \\
\text { Increased nutrient excretion } \\
\text { Increased fat excretion } \\
\text { Increased nutrient loss } \\
\text { Malabsorption } \\
\text { Increased protein excretion } \\
- \\
\text { Increased vitamin A excretion }\end{array}$ \\
\hline Complications & $\begin{array}{l}\text { Migration or } \\
\text { aggregation of } \\
\text { adult Ascaris } \\
\text { in intestine }\end{array}$ & $\begin{array}{l}\text { Intestinal obstruction } \\
\text { Intussusception } \\
\text { Invasion of bile duct } \\
\quad \text { (producing cholangitis } \\
\text { obstructive jaundice, } \\
\text { gallstones, or liver } \\
\text { abscesses) } \\
\text { Acute appendicitis } \\
\text { Acute pancreatitis } \\
\text { Intestinal perforation } \\
\text { Peritonitis } \\
\text { Upper respiratory tract obstruction } \\
\text { Volvulvus }\end{array}$ & $\begin{array}{l}\text { Life-threatening illnesses } \\
\text { which all decrease food } \\
\text { intake and may increase } \\
\text { nutrient requirements (due } \\
\text { due to fever) and nutrient } \\
\text { losses (due to diarrhoea) }\end{array}$ \\
\hline
\end{tabular}

Adapted from Stephenson \& Holland, 1987.

the larvae in the liver and lungs (Beaver et al. 1984). According to Pawlowski (1978), dying larvae are thought to do more harm to their hosts than the living ones. Larval migration can lead to the onset of pneumonitis, which can include asthma, cough, substernal pain, fever, skin rash and eosinophilia (Pawlowski \& Arfaa, 1984; Coles, 1985), a condition that can sometimes be fatal (Beaver \& Danaraj, 1958). In contrast, clinically evident pulmonary ascariasis is said to be relatively mild and short lived, lasting about five days (Gelphi \& Mustafa, 1967). Further population-based investigations of pulmonary ascariasis are required to define clearly its public health significance, in part because fever, when associated with respiratory disease, can increase urinary nitrogen loss and because respiratory infections are one of the major acute causes of death in young children in developing countries (Stephenson \& Holland, 1987).

Intestinal helminths, and $A$. lumbricoides especially, can provide particularly potent stimuli for the production of $\mathrm{IgE}$ antibody (Jarrett \& Miller, 1982). Using extracts derived from A. lumbricoides, bronchial challenge induced bronchoconstriction in clinically asthmatic children from helminth-endemic areas (Lynch et al. 1992a). Non-asthmatic children in such areas were later shown to respond significantly to bronochodilator inhalation, and this was reversible with anthelminthic treatment (Lynch et al. 1992b). A follow-up study of asthmatic patients in Venezuela, $23 \%$ of whom were infected at the beginning with $A$. lumbricoides, showed that regular anthelminthic treatment of 39 asthmatics with albendazole for a period of one year resulted in 
significant improvement up to two years (Lynch et al. 1997). After two years without treatment however, the severity of asthma reverted to the initial state. In contrast, no significant changes were observed in the 50 untreated patients over the same period of evaluation. IgE antibody levels and skin test positivity to Ascaris exhibited a 'tendency' towards an increase among those who had received anthelminthic treatment; Lynch et al. (1997) concluded that this might have been because asthmatic patients were being further exposed to Ascaris eggs over the duration of the study.

The presence of adult worms in the small intestine is thought to be generally well tolerated although large studies of symptoms in infected communities have not yet received sufficient attention (Table 2) (Stephenson \& Holland, 1987). Coles (1985) suggested that abdominal symptoms were the result of the host responding to toxins produced by the worms or to a peptide that causes the release of histamine or allergens resulting in immunopathology or a combination of these.

Most Ascaris infections are of a chronic form and are widely considered to significantly impair childhood nutrition, especially in areas where poor growth and ascariasis are common; the infection is most likely to affect bodily growth, fat absorption, vitamin A absorption, iodine absorption, lactose digestion, and protein absorption (Carrera, Nesheim \& Crompton, 1984; Stephenson \& Holland, 1987; Taren et al. 1987; Tomkins \& Watson, 1989; Thein Hlaing, 1993 ; Crompton, 1994; Curtale et al. 1994; Tanumihardjo et al. 1996; Furnee et al. 1997). Ascaris infection reduces appetite (Hadju et al. 1996 b; 1998), and the intestinal pathology that occurs in malnourished children includes villus atrophy and cellular infiltration of the lamina propria ('Tripathy et al. 1972). Furthermore, the causal association between ascariasis and protein-energy malnutrition is substantiated by the association between the intensity of Ascaris infection and the degree of malnutrition (Blumenthal \& Schultz, 1976; Thein Hlaing et al. $1991 a)$.

Because intestinal infections can lead to nutritional deficiencies, they can lower the immunity that is essential for the maintenance of innate resistance and the genetically constituted immune response that police parasites (Beisel, 1982; Puri \& Chandra, 1985). El-Araby, El-din \& Abdou (1984) found a relationship between ascariasis and impaired cellular response in Saudi Arabian children which would suggest that either pre-existing immunodeficiencies reduce resistance or that the presence of worms has an immunosuppressive effect.

A worm expulsion study carried out with 428 children (aged 4-10 years) in southeastern Madagascar revealed that in communities where children were predominantly stunted, A. lumbricoides did not always aggregate in the most malnourished or immunosuppressed children (Kightlinger, Seed \& Kightlinger, 1996). This, the authors concluded, suggested the independence of growth status and $A$. lumbricoides worm burden. In contrast, Thein Hlaing et al. (1991 a) showed that growth gains in children were dependent on the initial worm burden: those with higher initial worm burdens had lower increments in growth rates than those with lower worm burdens. Thus, children with recurrent heavy infections are at most risk of developing some permanent growth deficit due to protein-energy malnutrition; this latter finding is what one would expect in most communities based on decades of research on malnutrition and infections (Scrimshaw \& SanGiovanni, 1997).

Both adults and children experience acute lifethreatening ascariasis, in which intestinal obstructions and biliary complications predominate (Table 1) (Khuroo, Zargar \& Mahajan, 1990; Thein Hlaing et al. 1990; Chai et al. 1991; Chrungoo et al. 1992). De Silva et al. (1997a) estimated that 12 million acute cases occur each year with approximately 10000 deaths and that complications are very much more rare than faltering growth and are probably associated with higher worm burdens. These complications have a high case fatality rate (Pinus, 1985), are expensive to treat and are likely to cause frank malnutrition in those children who are not already malnourished when complications develop (Stephenson \& Holland, 1987).

De Silva et al. (1997b) concluded from an analysis of published reports on Ascaris-induced intestinal obstruction that it constitutes the commonest acute complication of ascariasis, accounting for almost $57 \%$ of all complications. Intestinal obstruction is most frequent in children less than 10 years of age, perhaps because the peak intensity of Ascaris is usually in the 5-10 year age group and because the narrow intestinal lumen diameter makes under 5 year olds more vulnerable to obstruction. The incidence was on the order of $0-0 \cdot 025$ cases per year per 1000 population in endemic regions, was non-linearly related to the prevalence of infection, and was associated with a mean case fatality rate of over $5 \%$.

\section{LONGITUDINAL FIELD STUDIES OF \\ A. LUMBRICOIDES-INDUCED MALNUTRITION \\ IN CHILDREN AND ITS IMPACT ON GROWTH}

One of the most important associations found in the studies reported below is that appetite improves after treatment of Ascaris infection in undernourished children. We expect that finding (see Table 6 showing depressed feed intakes in parasitic infections in various species, Malnutrition and Parasitic Helminth Infection, this volume), but food intakes are very difficult, labour intensive and expensive to 

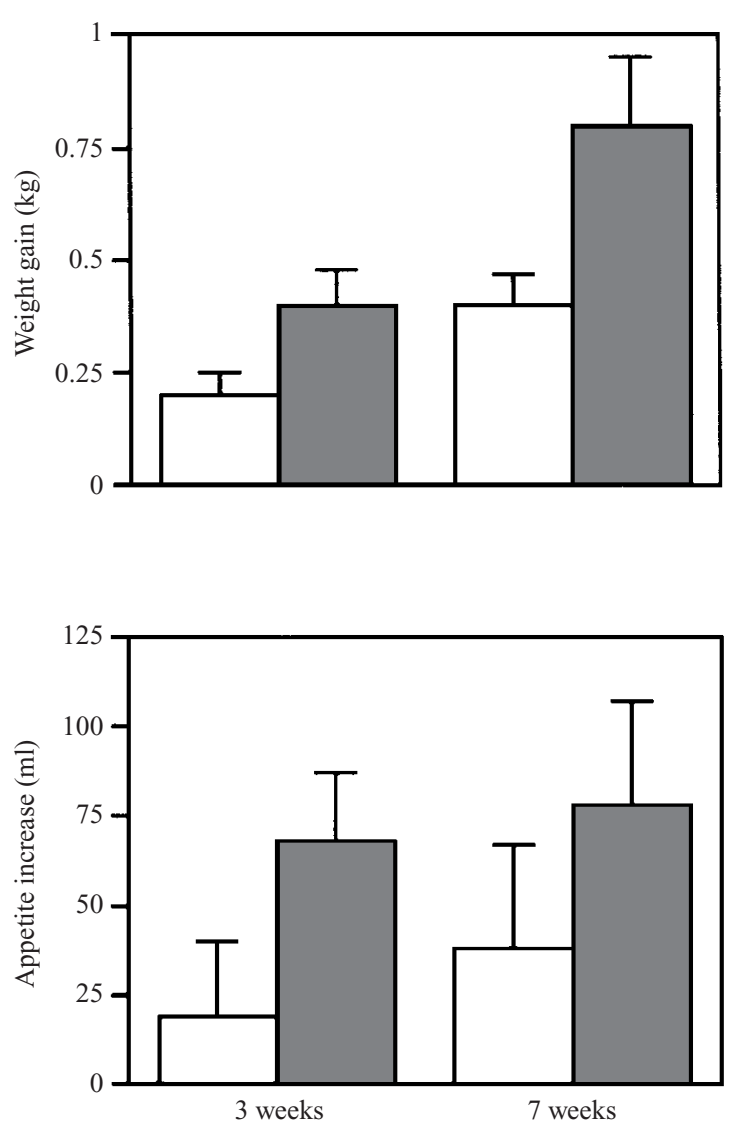

Fig. 2. Weight gain $(\mathrm{kg})$ and appetite increase $(\mathrm{ml})$ at 3 and 7 weeks after treatment for $A$. lumbricoides in Indonesian school boys in the pyrantel-treated (PR, shaded bars) and placebo (PL, open bars) groups. Values are means \pm s.E.M., $n=72$ at 3 weeks and 64 at 7 weeks. At baseline, there were no significant differences between groups in appetite and weight. At 3 weeks, increases in appetite and weight gain in the PR group were greater than in the PL group. (Group $t$-test $P$ for difference in weight gain in $\mathrm{kg}=0.53$ and for difference in appetite increase, $P=0 \cdot 04)$. At 7 weeks, increase in appetite in the $\mathrm{PR}$ group was not significantly greater than in the PL group $(P=0 \cdot 17)$ but weight gain was $(P=0 \cdot 02)$. Source: Hadju et al. $(1996 a)$. Reprinted with permission from Parasitology (Cambridge University Press).

quantify precisely in free-living humans, especially without influencing what is eaten and are rarely measured in parasitized children. The study that demonstrates this relationship most definitively for ascariasis in children may be that of Hadju et al. $(1996 a)$, in which 6-10 year old Indonesian school boys were given pyantel pamoate (which has little or no effect on Trichuris trichiura) for $A$. lumbricoides infection ( $86 \%$ baseline prevalence); they ate significantly more of a mid-day snack offered $a d l i b$. at school at 3 weeks after treatment. It was reported that their appetites were significantly better than placebo boys both 3 and 7 weeks after treatment, and they also had gained significantly more weight 7 weeks after treatment (Fig. 2). A related study, also in Indonesian schoolboys, found increased free play activity as measured by Caltrac ${ }^{\mathrm{TM}}$ accelerometers, in addition to improved appetite and growth (heightfor-age $\mathrm{Z}$-scores and mid upper arm circumference) 6 months after treatment for $A$. lumbricoides and $T$. trichiura infections with albendazole (Hadju et al. 1998). These findings are important for mental and social as well as physical development because children learn more when they are healthy and have the energy to be active. Ten intervention studies which examined the extent to which growth rates improve in undernourished Ascaris-infected children following anthelminthic chemotherapy are described below.

\section{Study design, country, population, intervention, duration, and measurements}

All ten studies reviewed here on childhood malnutrition were undertaken in developing countries in Asia and Africa (Table 3). The study populations were pre-school and school age children. Apart from one Kenyan study in which growth was examined in Ascaris-infected and non-infected groups (Stephenson et al. $1980 a$ ), the children were allocated at random to either control or treatment groups. One study however, randomised children and allocated them to one of three groups: placebo, one-dose treatment and two-dose treatments (Stephenson et al. 1993a). The anthelminthic drugs tetramisole, levamisole, albendazole and pyrantel pamoate were used in these studies; dosing frequencies used are shown in Table 3. Albonico et al. (1999) give a detailed profile of these and other anthelminthic drugs currently used to treat Ascaris infection. The study duration ranged from a minimum of 7 weeks in Indonesia (Hadju et al. 1996a) to two years in Myanmar (Thein Hlaing et al. 1991a) (Table 3). The prevalences of the various intestinal parasites observed in these intervention studies at baseline are shown in Table 4. Among the body growth indicators also measured in these studies were weight, height, arm circumference, triceps skinfold thickness, and subscapular skinfold thickness, using various reference standard populations. Details of the numbers of subjects assigned to the control and treatment groups feature in Table 5.

\section{Statistical analyses}

Most studies used paired or group Student's $t$-tests to test for comparability of control and treatment groups at baseline and to test for significant differences in growth rates between treatment and control groups following anthelminthic intervention (Stephenson et al. 1980a , 1989, 1993b; Thein Hlaing et al. 1991 a ; Adams et al. 1994; Hadju et al. $1996 a$, 1998). This same relationship was also examined using Chi-square analysis (Gupta et al. 1977; Gupta, 1985), analysis of variance (Stephenson et al. 1993a) and multiple regression (Willett, Kilama \& Kihamia, 
Table 3. Longitudinal field studies of A. lumbricoides-induced malnutrition in children: country of study, anthelminthic drug regime employed, and duration of follow-up

\begin{tabular}{|c|c|c|c|c|}
\hline $\begin{array}{l}\text { Duration } \\
\text { follow-up } \\
\text { (Months) }\end{array}$ & Country of study & Drug used & Frequency & Reference \\
\hline 12 & India & Tetramisole & 3 times 4-monthly & Gupta et al. (1977), Gupta (1985) \\
\hline 12 & Tanzania & Levamisole & 4 times 3-monthly & Willett et al. (1979) \\
\hline $3 \cdot 5$ & Kenya & Levamisole & Single & Stephenson et al. $(1980 a)$ \\
\hline 6 & Kenya & Albendazole & Single & Stephenson et al. (1989) \\
\hline 24 & Myanmar & Levamisole & 8 times 4-monthly & Thein Hlaing et al. (1991) \\
\hline $8 \cdot 2$ & Kenya & Albendazole & Single, double & Stephenson et al. $(1993 a)$ \\
\hline 4 & Kenya & Albendazole & Single & Stephenson et al. $(1993 b)$ \\
\hline $2 \cdot 25$ & Kenya & Albendazole & Single & Adams et al. (1994) \\
\hline $1 \cdot 75$ & Indonesia & Pyrantel pamoate & Single & Hadju et al. (1996a) \\
\hline 6 & Indonesia & Albendazole & Single & Hadju et al. (1998) \\
\hline
\end{tabular}

Table 4. Baseline prevalence of major intestinal parasites in randomized intervention study areas

\begin{tabular}{|c|c|c|c|c|c|}
\hline Reference & Group & $n$ & $\begin{array}{l}\text { A. lumbricoides } \\
\text { prevalence } \\
(\%)\end{array}$ & $\begin{array}{l}\text { T. trichiura } \\
\text { prevalence } \\
(\%)\end{array}$ & $\begin{array}{l}\text { Hookworm } \\
\text { prevalence } \\
(\%)\end{array}$ \\
\hline \multirow[t]{2}{*}{ Gupta et al. (1977); Gupta (1985) } & I & 143 & 40 & - & - \\
\hline & $\mathrm{N}$ & 98 & 46 & - & - \\
\hline \multirow[t]{2}{*}{ Willett et al. (1979) } & I & 166 & 51 & - & 10 \\
\hline & $\mathrm{N}$ & 175 & 55 & - & 12 \\
\hline \multirow[t]{2}{*}{ Stephenson et al. (1989) } & I & 78 & 44 & 98 & 95 \\
\hline & $\mathrm{N}$ & 72 & 54 & 97 & 79 \\
\hline \multirow[t]{2}{*}{ Thein Hlaing et al. (1991a) } & I & 595 & 81 & 5 & 2 \\
\hline & $\mathrm{N}$ & 611 & 83 & 7 & 1 \\
\hline \multirow[t]{3}{*}{ Stephenson et al. $(1993 a)$} & $\mathrm{I}-1 \mathrm{X}$ & 96 & 35 & 90 & 85 \\
\hline & $\mathrm{I}-2 \mathrm{X}$ & 95 & 26 & 81 & 86 \\
\hline & $\mathrm{N}$ & 93 & 32 & 92 & 88 \\
\hline \multirow[t]{2}{*}{ Stephenson et al. $(1993 b)$} & I & 27 & 44 & 96 & 96 \\
\hline & $\mathrm{N}$ & 26 & 38 & 100 & 96 \\
\hline \multirow[t]{2}{*}{ Adams et al. 1994} & I & 28 & 32 & 79 & 93 \\
\hline & $\mathrm{N}$ & 27 & 26 & 89 & 93 \\
\hline \multirow[t]{2}{*}{ Hadju et al. (1998) } & I & 86 & 93 & 97 & - \\
\hline & $\mathrm{N}$ & 43 & 95 & 98 & - \\
\hline \multirow{2}{*}{ Hadju et al. (1996a) } & I & 36 & 89 & 100 & - \\
\hline & $\mathrm{N}$ & 36 & 86 & 100 & - \\
\hline
\end{tabular}

I, Intervention group; I-1X, Intervention 1 dose; I-2X, Intervention 2 doses; N, Non-intervention group.

1979; Stephenson et al. $1980 a, 1989,1993 a, b)$. Among the identified confounding variables were socio-economic factors, health and nutritional status and the presence of other helminth infections.

\section{RESULTS}

Weight and/or weight-for-age were examined as a body growth indicator in all 10 studies. Eight of 9 using weight in $\mathrm{kg}$ for hypothesis testing found a statistically significant improvement in weight after treatment; in the Tanzanian study, the significant gain occurred in initially Ascaris-positive children in the treatment group vs. those given a placebo (Willett et al. (1979). Seven studies (Gupta et al. 1977; Gupta, 1985; Stephenson et al. 1980a, 1989, $1993 a, b$; Hadju et al. 1996a) also calculated percentage weight-for-age and all of them apart from Stephenson et al. $(1980 a$; borderline $P<0 \cdot 10)$ and Hadju et al. $1998(P>0 \cdot 10)$ showed statistically significant improvements in intervention groups compared to non-intervention groups (Table 5). 
Table 5. Difference in increments in growth following treatment between intervention and non-intervention groups for longitudinal field studies of ascariasis in children: weight; $\%$ weight-for-age, height, $\%$ height-for-age, $\%$ weight-for-height, $\%$ of median or $Z$-scores

\begin{tabular}{|c|c|c|c|c|c|c|c|c|c|c|c|c|c|c|c|c|c|}
\hline \multirow[b]{2}{*}{ References } & \multirow[b]{2}{*}{ Group } & \multirow[b]{2}{*}{$n$} & \multicolumn{15}{|c|}{ Increment in weight, height and weight for height } \\
\hline & & & $\begin{array}{l}\text { Weight } \\
(\mathrm{kg})\end{array}$ & Diff. & $P$ & $\begin{array}{l}\text { Weight for } \\
\text { age (\% or } \\
Z \text {-score) }\end{array}$ & Diff. & $P$ & $\begin{array}{l}\text { Height } \\
(\mathrm{cm})\end{array}$ & Diff. & $P$ & $\begin{array}{l}\text { Height for } \\
\text { age }(\% \text { or } \\
Z \text {-score) }\end{array}$ & Diff. & $P$ & $\begin{array}{l}\text { Weight for } \\
\text { height ( } \% \text { or } \\
Z \text {-score) }\end{array}$ & Diff. & $P$ \\
\hline Gupta et al. $(1977,1985)$ & I & $\begin{array}{l}74 \\
80\end{array}$ & - & & & $\begin{array}{r}3 \cdot 5 \\
-0.3\end{array}$ & $-3 \cdot 5$ & $<0.001$ & - & & & - & & & - & & \\
\hline Willett et al. (1979) & I & $\begin{array}{l}273 \\
273\end{array}$ & $\begin{array}{l}2.08 \\
1.92\end{array}$ & $0 \cdot 2$ & $<0 \cdot 06$ & - & & & - & & & - & & & - & & \\
\hline Stephenson et al. $(1980 a)$ & I & $\begin{array}{r}61 \\
125\end{array}$ & $\begin{array}{l}0.07 \pm 0.4 \\
0.05 \pm 0.5\end{array}$ & $0 \cdot 2$ & $<0.05$ & $\begin{array}{l}1 \cdot 6 \pm 2 \cdot 7 \\
0 \cdot 7 \pm 3 \cdot 1\end{array}$ & 0.9 & $<0 \cdot 10$ & - & & & - & & & - & & \\
\hline Stephenson et al. (1989) & I & $\begin{array}{l}78 \\
72\end{array}$ & $\begin{array}{l}2 \cdot 1 \pm 0 \cdot 10 \\
0 \cdot 8 \pm 0 \cdot 10\end{array}$ & $1 \cdot 3$ & $<0.0002$ & $\begin{array}{r}1 \cdot 8 \pm 0 \cdot 29 \\
-2 \cdot 7 \pm 0 \cdot 34\end{array}$ & $4 \cdot 5$ & $<0.0002$ & $\begin{array}{l}2 \cdot 2 \pm 0 \cdot 10 \\
1 \cdot 8 \pm 0 \cdot 29\end{array}$ & 0.6 & $<0.0002$ & $\begin{array}{l}-0.2 \pm 0.06 \\
-0.7 \pm 0.08\end{array}$ & 0.5 & $<0.0002$ & & & \\
\hline Thein Hlaing et al. (1991 a) & $\mathrm{I}_{\mathrm{N}}$ & $\begin{array}{l}210 \\
205\end{array}$ & $\begin{array}{l}3 \cdot 6 \pm 1 \cdot 28 \\
2 \cdot 6 \pm 1 \cdot 04\end{array}$ & $0 \cdot 9$ & $<0.001$ & & & & $\begin{array}{l}11 \cdot 3 \pm 2 \cdot 04 \\
10 \cdot 3 \pm 1 \cdot 77\end{array}$ & 0.9 & $<0.001$ & & & & & & \\
\hline Stephenson et al. (1993a) & $\begin{array}{l}I-1 X \\
I-2 X \\
N\end{array}$ & $\begin{array}{l}96 \\
95 \\
93\end{array}$ & $\begin{array}{l}3 \cdot 3 \pm 0 \cdot 18 \\
3 \cdot 1 \pm 0 \cdot 14 \\
2 \cdot 2 \pm 0 \cdot 12\end{array}$ & $\begin{array}{l}1 \cdot 1 \\
0 \cdot 9\end{array}$ & $<0 \cdot 0001 *$ & $\begin{array}{r}1 \cdot 9 \pm 0 \cdot 36 \\
1 \cdot 3 \pm 0 \cdot 30 \\
-1 \cdot 4 \pm 0 \cdot 28\end{array}$ & $\begin{array}{l}3 \cdot 3 \\
2 \cdot 7\end{array}$ & $<0.0001 *$ & $\begin{array}{l}3 \cdot 8 \pm 0 \cdot 12 \\
3 \cdot 6 \pm 0 \cdot 11 \\
3 \cdot 7 \pm 0 \cdot 12\end{array}$ & $\begin{array}{r}0 \cdot 1 \\
-0.1\end{array}$ & NS & $\begin{array}{l}-0 \cdot 2 \pm 0 \cdot 08 \\
-0 \cdot 3 \pm 0 \cdot 08 \\
-0 \cdot 4 \pm 0 \cdot 07\end{array}$ & $\begin{array}{l}0 \cdot 2 \\
0 \cdot 1\end{array}$ & NS & $\begin{array}{l}2 \cdot 8 \pm 0 \cdot 36 \\
2 \cdot 6 \pm 0 \cdot 35 \\
0 \cdot 3 \pm 0 \cdot 30\end{array}$ & $\begin{array}{l}3 \cdot 1 \\
2 \cdot 9\end{array}$ & $<0.0001 *$ \\
\hline Stephenson et al. (1993b) & I & $\begin{array}{l}27 \\
26\end{array}$ & $\begin{array}{l}1 \cdot 6 \pm 0 \cdot 15 \\
0 \cdot 6 \pm 0 \cdot 08\end{array}$ & $1 \cdot 0$ & $<0.0002$ & $\begin{array}{r}1 \cdot 0 \pm 0 \cdot 42 \\
-2 \cdot 0 \pm 0 \cdot 24\end{array}$ & $3 \cdot 0$ & $<0.0002$ & $\begin{array}{l}2 \cdot 0 \pm 0 \cdot 19 \\
1 \cdot 4 \pm 0 \cdot 08\end{array}$ & 0.6 & $<0.003$ & $\begin{array}{l}-0 \cdot 1 \pm 0 \cdot 13 \\
-0 \cdot 6 \pm 0 \cdot 28\end{array}$ & 0.5 & $<0.0015$ & $\begin{array}{r}1 \cdot 6 \pm 0.49 \\
-0 \cdot 6 \pm 0.28\end{array}$ & $2 \cdot 2$ & $<0.0002$ \\
\hline Adams et al. (1994) & $\begin{array}{l}\mathrm{I} \\
\mathrm{N}\end{array}$ & $\begin{array}{l}28 \\
27\end{array}$ & $\begin{array}{l}1 \cdot 0 \pm 0 \cdot 06 \\
0 \cdot 3 \pm 0 \cdot 10\end{array}$ & 0.7 & $<0.0002$ & $\begin{array}{l}0 \cdot 30 \pm 0 \cdot 024 Z \\
0 \cdot 08 \pm 0 \cdot 034 Z\end{array}$ & 0.22 & $<0.0002$ & $\begin{array}{l}0 \cdot 9 \pm 0 \cdot 10 \\
0 \cdot 8 \pm 0 \cdot 11\end{array}$ & 0.1 & NS & $\begin{array}{l}0 \cdot 16 \pm 0 \cdot 017 Z \\
0 \cdot 16 \pm 0 \cdot 023 Z\end{array}$ & $0 \cdot 00$ & NS & $\begin{array}{r}0 \cdot 33 \pm 0.036 \mathrm{Z} \\
-0.03 \pm 0.060 \mathrm{Z}\end{array}$ & $0 \cdot 3$ & $<0.0002$ \\
\hline Hadju et al. (1996a) & $\begin{array}{l}\text { I } \\
\mathrm{N}\end{array}$ & $\begin{array}{l}34 \\
30\end{array}$ & $\begin{array}{l}0 \cdot 8 \pm 0 \cdot 9 \\
0 \cdot 4 \pm 0 \cdot 4\end{array}$ & 0.4 & $<0 \cdot 02$ & $\begin{array}{r}1 \cdot 5 \pm 3 \cdot 4 \\
-0 \cdot 2 \pm 0 \cdot 3\end{array}$ & 1.7 & $<0.02$ & - & & & - & & & - & & \\
\hline Hadju et al. (1998) & $\begin{array}{l}\mathrm{I} \\
\mathrm{N}\end{array}$ & $\begin{array}{l}86 \\
43\end{array}$ & $\begin{array}{l}1.08 \pm 0.6 \\
1 \cdot 09 \pm 0.8\end{array}$ & 0.01 & NS & $\begin{array}{l}0 \cdot 05 \pm 0 \cdot 2 Z \\
0 \cdot 06 \pm 0 \cdot 2 Z\end{array}$ & 0.01 & NS & $\begin{array}{l}3 \cdot 54 \pm 0.9 \\
3 \cdot 44 \pm 0.8\end{array}$ & $0 \cdot 10$ & NS & $\begin{array}{l}0 \cdot 16 \pm 0 \cdot 1 Z \\
0 \cdot 10 \pm 0 \cdot 2 Z\end{array}$ & 0.06 & $<0.03$ & $\begin{array}{l}-0 \cdot 13 \pm 0 \cdot 3 Z \\
-0 \cdot 10 \pm 0 \cdot 3 Z\end{array}$ & 0.03 & NS \\
\hline
\end{tabular}

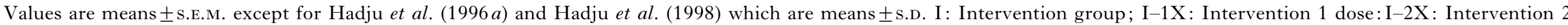

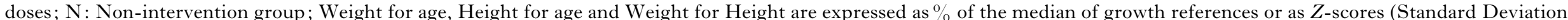
units). Diff.: Difference. $P, t$-test significances value; *, ANOVA Tukey honestly significant difference test significance value; NS, not statistically significant. 


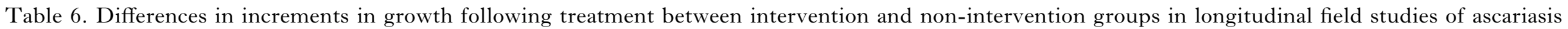
in children: triceps skinfold thickness, triceps skinfold thickness for age (\%), arm circumference, arm circumference for age ( $\%)$, subscapular skinfold thickness and subcapular skinfold thickeness for age, $\%$ median or $Z$-scores

\begin{tabular}{|c|c|c|c|c|c|c|c|c|c|c|c|c|c|c|c|c|c|c|c|c|}
\hline Reference & Group & $n$ & $\begin{array}{l}\text { Triceps } \\
\text { skinfold } \\
\text { thickness } \\
\text { (mm) }\end{array}$ & Diff. & $P$ & $\begin{array}{l}\text { Triceps } \\
\text { skinfold } \\
\text { thickness } \\
\text { for age } \\
\text { (\% or } \\
Z \text {-score) }\end{array}$ & Diff. & $P$ & $\begin{array}{l}\text { Arm } \\
\text { circum. } \\
(\mathrm{cm})\end{array}$ & Diff. & $P$ & $\begin{array}{l}\text { Arm } \\
\text { circum. } \\
\text { for age } \\
(\% \text { or } \\
Z \text {-score })\end{array}$ & Diff. & $P$ & $\begin{array}{l}\text { Subscapular } \\
\text { skinfold } \\
\text { thickness } \\
(\mathrm{mm})\end{array}$ & Diff. & $P$ & $\begin{array}{l}\text { Subscapular } \\
\text { skinfold } \\
\text { thickness } \\
\text { for age } \\
\text { (\% or } \\
Z \text {-score) }\end{array}$ & Diff. & $P$ \\
\hline Stephenson et al. (1980a) & $\begin{array}{l}\mathrm{I} \\
\mathrm{N}\end{array}$ & $\begin{array}{r}61 \\
125\end{array}$ & $\begin{array}{r}2 \cdot 0 \pm 0 \cdot 9 \\
-1 \cdot 1 \pm 1 \cdot 2\end{array}$ & $2 \cdot 9$ & $<0.0005$ & $\begin{array}{c}21 \cdot 7 \pm 9 \cdot 7 \\
-10 \cdot 4 \pm 13 \cdot 4\end{array}$ & $32 \cdot 1$ & $<0.0005$ & $\begin{array}{l}- \\
-\end{array}$ & & & $\begin{array}{l}- \\
-\end{array}$ & - & & - & - & - & & & \\
\hline Stephenson et al. (1989) & $\begin{array}{l}\mathrm{I} \\
\mathrm{N}\end{array}$ & $\begin{array}{l}78 \\
72\end{array}$ & $\begin{array}{r}1 \cdot 0 \pm 0 \cdot 08 \\
-0 \cdot 2 \pm 0 \cdot 08\end{array}$ & $1 \cdot 2$ & $<0.0002$ & $\begin{array}{r}9 \cdot 4 \pm 0 \cdot 87 \\
-3 \cdot 3 \pm 0 \cdot 77\end{array}$ & $12 \cdot 7$ & $<0.0002$ & $\begin{array}{l}0 \cdot 7 \pm 0.05 \\
0 \cdot 2 \pm 0.05\end{array}$ & 0.5 & $<0.0002$ & $\begin{array}{r}1 \cdot 7 \pm 0 \cdot 24 \\
-1 \cdot 2 \pm 0 \cdot 28\end{array}$ & $2 \cdot 9$ & $<0.0002$ & $\begin{array}{r}0 \cdot 9 \pm 0 \cdot 07 \\
-0 \cdot 3 \pm 0 \cdot 08\end{array}$ & $1 \cdot 2$ & $<0.0002$ & $\begin{array}{r}11 \cdot 8 \pm 1 \cdot 32 \\
-9 \cdot 5 \pm 0 \cdot 08\end{array}$ & $21 \cdot 3$ & $<0.0002$ \\
\hline Stephenson et al. (1993a) & $\begin{array}{l}\mathrm{I}-1 \mathrm{X} \\
\mathrm{I}-2 \mathrm{X} \\
\mathrm{N}\end{array}$ & $\begin{array}{l}96 \\
95 \\
93\end{array}$ & $\begin{array}{l}2 \cdot 0 \pm 0 \cdot 11 \\
2 \cdot 0 \pm 0 \cdot 12 \\
0 \cdot 2 \pm 0 \cdot 08\end{array}$ & $\begin{array}{l}1 \cdot 8 \\
1 \cdot 8\end{array}$ & $<0 \cdot 0001 *$ & $\begin{array}{r}17 \cdot 0 \pm 0 \cdot 98 \\
17 \cdot 1 \pm 0 \cdot 94 \\
0 \cdot 5 \pm 0 \cdot 79\end{array}$ & $\begin{array}{l}16 \cdot 5 \\
16 \cdot 5\end{array}$ & $<0.0005^{*}$ & $\begin{array}{l}0 \cdot 8 \pm 0.05 \\
0 \cdot 7 \pm 0.05 \\
0 \cdot 3 \pm 0.04\end{array}$ & $\begin{array}{l}0 \cdot 5 \\
0 \cdot 4\end{array}$ & $<0 \cdot 0001 *$ & $\begin{array}{l}0 \cdot 8 \pm 0.05 \\
0 \cdot 7 \pm 0.05 \\
0 \cdot 3 \pm 0.04\end{array}$ & $\begin{array}{l}0 \cdot 5 \\
0 \cdot 4\end{array}$ & $<0 \cdot 0001 *$ & $\begin{array}{l}1 \cdot 8 \pm 0.09 \\
1 \cdot 9 \pm 0 \cdot 11 \\
0 \cdot 4 \pm 0.08\end{array}$ & $\begin{array}{l}1 \cdot 4 \\
1 \cdot 5\end{array}$ & $<0 \cdot 0001 *$ & $\begin{array}{r}23 \cdot 7 \pm 1 \cdot 19 \\
26 \cdot 2 \pm 1 \cdot 53 \\
2 \cdot 4 \pm 1 \cdot 25\end{array}$ & $\begin{array}{l}21 \cdot 3 \\
23 \cdot 8\end{array}$ & $<0.0001 *$ \\
\hline Stephenson et al. (1993b) & $\begin{array}{l}\mathrm{I} \\
\mathrm{N}\end{array}$ & $\begin{array}{l}27 \\
26\end{array}$ & $\begin{array}{r}1 \cdot 0 \pm 0 \cdot 09 \\
-0 \cdot 0 \pm 0 \cdot 10\end{array}$ & $1 \cdot 0$ & $<0.0002$ & $\begin{array}{r}10 \cdot 2 \pm 1 \cdot 04 \\
-1 \cdot 4 \pm 1 \cdot 19\end{array}$ & $11 \cdot 6$ & $<0.0002$ & $\begin{array}{r}0 \cdot 3 \pm 0 \cdot 06 \\
-0 \cdot 0 \pm 0 \cdot 05\end{array}$ & $0 \cdot 3$ & $<0.0002$ & $\begin{array}{l}-0 \cdot 0 \pm 0 \cdot 33 \\
-1 \cdot 5 \pm 0.23\end{array}$ & 1.5 & $<0.0005$ & $\begin{array}{l}1 \cdot 0 \pm 0.09 \\
0 \cdot 0 \pm 0 \cdot 07\end{array}$ & $1 \cdot 0$ & $<0.0002$ & $\begin{array}{r}17 \cdot 1 \pm 1 \cdot 67 \\
-1 \cdot 0 \pm 0 \cdot 36\end{array}$ & $18 \cdot 1$ & $<0.0002$ \\
\hline Adams et al. (1994) & $\begin{array}{l}\mathrm{I} \\
\mathrm{N}\end{array}$ & $\begin{array}{l}28 \\
27\end{array}$ & $\begin{array}{l}1 \cdot 0 \pm 0 \cdot 13 \\
0 \cdot 2 \pm 0 \cdot 09\end{array}$ & $0 \cdot 8$ & $<0.0002$ & $\begin{array}{l}0 \cdot 37 \pm 0 \cdot 051 \mathrm{Z} \\
0 \cdot 09 \pm 0 \cdot 035 \mathrm{Z}\end{array}$ & $0 \cdot 28$ & $<0 \cdot 0002$ & $\begin{array}{l}0 \cdot 6 \pm 0.07 \\
0 \cdot 3 \pm 0.05\end{array}$ & $0 \cdot 3$ & $<0.0002$ & $\begin{array}{l}0 \cdot 40 \pm 0 \cdot 045 \mathrm{Z} \\
0 \cdot 16 \pm 0 \cdot 037 \mathrm{Z}\end{array}$ & $0 \cdot 24$ & $<0.0002$ & $\begin{array}{l}0 \cdot 9 \pm 0 \cdot 10 \\
0 \cdot 0 \pm 0 \cdot 18\end{array}$ & 0.8 & $<0.0002$ & $\begin{array}{l}0.82 \pm 0 \cdot 092 Z \\
0 \cdot 04 \pm 0 \cdot 129 Z\end{array}$ & 0.78 & $<0.0002$ \\
\hline Hadju et al. (1998) & $\begin{array}{l}\mathrm{I} \\
\mathrm{N}\end{array}$ & $\begin{array}{l}86 \\
43\end{array}$ & $\begin{array}{l}0 \cdot 98 \pm 1 \cdot 5 \\
0 \cdot 70 \pm 1 \cdot 3\end{array}$ & $0 \cdot 28$ & $0 \cdot 15$ & $\begin{array}{l}0 \cdot 22 \pm 0 \cdot 6 \mathrm{Z} \\
0 \cdot 19 \pm 0 \cdot 5 \mathrm{Z}\end{array}$ & 0.03 & $0 \cdot 30$ & $\begin{array}{l}0 \cdot 62 \pm 0 \cdot 6 \\
0 \cdot 33 \pm 0 \cdot 7\end{array}$ & $0 \cdot 29$ & $<0.01$ & $\begin{array}{r}0 \cdot 07 \pm 0 \cdot 4 \mathrm{Z} \\
-0 \cdot 07 \pm 0 \cdot 5 \mathrm{Z}\end{array}$ & 0.14 & $<0.02$ & $\begin{array}{l}- \\
-\end{array}$ & & & $\begin{array}{l}- \\
-\end{array}$ & & \\
\hline
\end{tabular}

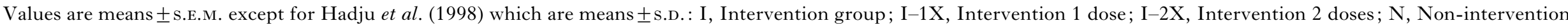

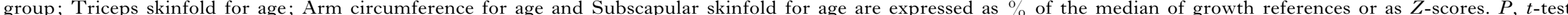
significance value; ANOVA Tukey honestly significant difference test significance value; NS, Not statistically significant; Diff. : Difference. 
Stephenson et al. (1989, 1993a, 1993b), Thein Hlaing et al. (1991 a), Adams et al. (1994) and Hadju et al. (1998) measured height in their studies and it was only Stephenson et al. (1993a) and Adams et al. (1994) who did not find a statistically significant result. Three of the five studies which examined height for age ( $\%$ of median or $\mathrm{Z}$-scores) found significant improvement at follow-up. Four studies, Stephenson et al. (1993a,b), calculated weight-forheight; 3 of those reported statistically significant differences (Table 5). Stephenson et al. (1989; $1993 a, b)$, Adams et al. (1994) and Hadju et al. (1998) assessed arm circumference and arm circumference-for-age in their studies and found them to be significantly improved after treatment (Table $6)$.

When triceps skinfold thickness was investigated (Stephenson et al. $1980 a$; 1989; $1993 a, b$; Adams et al. 1994; Hadju et al. 1998), it was found in 4 of 5 studies to be highly statistically significantly associated with improved growth in children. Subscapular skinfold thickness was measured by Stephenson et al. $(1989 ; 1993 a, b)$ and Adams et al. (1994) and was shown to be highly statistically significant in each case $(P<0 \cdot 0005$, Table 6). A multiple regression analysis by Stephenson et al. (1980a) also revealed that $A$. lumbricoides infection was by far the most important of 37 possible health, nutritional and socio-economic variables in explaining the differences in skinfold thickness observed both before and after treatment. In three studies where children were found to be infected with a variety of helminths, decreases in intensities, including those of Ascaris, Trichuris trichiura and hookworm, were significant predictors of growth improvement in multiple regression analyses (Stephenson et al. 1989; $1993 a, b)$.

A. LUMBRICOIDES AND COGNITIVE

PERFORMANCE IN CHILDREN

In 1993, Connolly \& Kvalsvig stated there was a good deal of evidence linking malnutrition with the impairment of cognitive function but that there was a paucity of data indicating a causal link between it and parasitic illness. However, some studies have implicated Ascaris infections in impairments of mental processing in some school children.

Kvalsvig, Cooppan \& Connolly (1991), for example, carried out two studies in Natal, near Durban in South Africa to consider the link between infection, malnutrition and impaired cognitive function. Of the 276 children in the survey, some were infected with a combination of $A$. lumbricoides, Schistosoma spp., T. trichiura, and hookworm. In the first study, children were given tests of information processing and perceptual speed before and after treatment with a single dose of $500 \mathrm{mg}$ of mebe- ndazole. The pattern of results was consistent with the hypothesis that parasitic infection combines with nutritional deficits to impair the efficiency of $\operatorname{cog}$ nitive processes. However, there were some confounding variables, and the single drug treatment reduced but did not eliminate the parasites. A second study removed the confounding variables of age and nutrition and employed a more comprehensive drug treatment programme. A memory task and a test of sustained attention were administered. Parasitic status showed a significant association with poor performance on the attention task, but no association was observed with educational attainment or memory function.

Later, Watkins, Cruz \& Pollitt (1996) carried out a similar study of the effects of deworming with albendazole on indicators of school performance in 246 rural Guatemalan children, aged 7-12 years. Ninety-one percent of children harboured A. lumbricoides, and $82 \%$ had $T$. trichiura; they were randomly assigned to treatment and control groups at 0 and 12 weeks in a double blind study. Albendazole successfully reduced the prevalence of Ascaris but it was less successful against Trichuris at the $400 \mathrm{mg}$ dosage used. The treated children were largely free of Ascaris for at least 6 months, but during that period, no improvement in reading, vocabulary or attendance was observed.

More recently, Hadidjaja et al. (1998) conducted a study with 336 children (aged 6-8 years) in northern Jakarta, Indonesia among whom the prevalence of $A$. lumbricoides was $58 \%$. The objective of the study was to investigate the effect of treatment with mebendazole and health education on nutritional status and cognitive function. The children were divided into five groups: Group 1 was given the anthelminthic mebendazole, group 2 was provided with health education, group 3 was given both the anthelminthic and health education, group 4 was given a placebo, and group 5 consisted of children whose stools tested negative for Ascaris eggs. After intervention, a mean prevalence of $41 \%$ was found. Approximately $80 \%$ of the children showed good scores for nutritional status in the pre- and posttreatment data, and only a small percentage $(0 \cdot 9-16 \cdot 2 \%)$ showed mild or moderate malnutrition. No significant difference in pre- and post-treatment nutritional status was observed. However, cognitive test results indicated that the group treated with mebendazole showed significant improvements in learning ability, concentration and eye-hand coordination 5 months post-treatment.

\section{A. LUMBRICOIDES AND ANTHELMINTHIC CONTROL STRATEGIES}

\section{Approaches to control strategies}

The main elements in planning anthelminthic con- 
trol programmes are epidemiology, targets, chemotherapy, health education, sanitation, monitoring and evaluation (Albonico et al. 1999). According to the WHO (1985b), these elements should be integrated into the prevailing system of primary health care and must be based on multisectoral collaboration. Control programmes cannot be planned, implemented or sustained without recent and reliable information on the infections of interest, the people at risk and the associated morbidity. Geographical distribution, seasonality and re-infection rates of helminth infection are also crucial elements to consider in well-planned control programmes (Albonico et al. 1999).

Control programmes based on sanitation aim to reduce or interrupt transmission, prevent re-infection and gradually to reduce worm loads (Wong \& Bundy, 1990). However, despite the well-recognized role of effective sanitation in preventing transmission of intestinal helminths, the impact of improved water supply and sanitation intervention has not been well studied. In heavily or frequently contaminated areas, one can expect decades to elapse before improved sanitation by itself measurably decreases A. lumbricoides infection (Huttly, 1990). However, the use and availability of latrines combined with periodic chemotherapy enhances the reduction in intensity of helminth infection and lengthens the time-span of re-infection both in research studies (Esrey et al. 1991) and control programmes (Albonico et al. 1999). For example, one study from St. Lucia reported that prevalence of infection with $A$. lumbricoides and $T$. trichiura in children was significantly lower in areas with improved sanitary conditions, as was the re-infection rate 6 months after chemotherapy (Henry, 1988).

In the long term, the prevention and control of ascariasis will be dependent upon economic development with consequent improvements in water supplies, sanitation and health education. Due to the scarcity of resources in most developing countries, the emphasis is currently on developing more costeffective approaches to control using available chemotherapeutic agents as a short-term goal (Savioli, Bundy \& Tomkins, 1992). The optimal choice of a chemotherapy control strategy is also based upon a number of factors including ease of administration and acceptability to the target population (Holland $e t$ al. 1996a). Current helminth control programmes are focused on reducing infection load and transmission potential in order to reduce morbidity and avoid mortality associated with the disease rather than to eradicate infection (Gilles, 1985; WHO, 1987). Repeated treatment ensures that even if reinfection occurs, intensity is maintained below the level associated with morbidity (Savioli et al. 1992).

Crompton (1994) describes in detail four main practical and feasible approaches to developing control strategies for ascariasis: (1) A mathematical approach that attempts to identify the gaps in knowledge of how $A$. lumbricoides persists with such stability in human communities; (2) The integration of family planning, nutrition and parasite control programmes, as exemplified by the broad-reaching activities of the Japanese Organisation for International Co-operation in Family Planning and the Asian Parasite Control Organisation; (3) The establishment of primary health care infrastructures in developing countries, whose populations must endure most of the morbidity associated with ascariasis; (4) Anthelminthic drugs that provide the most rapid means of reducing the intensity of infection and thereby reducing morbidity to more tolerable levels.

Epidemiological evidence suggests that anthelminthic chemotherapy can be used in one of three ways in community control programmes: universal, targeted or selective treatment (WHO, 1996b), defined as follows by Anderson (1989) and Albonico et al. (1999). (1) Offering universal treatment to all individuals in an area of high endemicity irrespective of the age, gender, worm burdens or other social characteristic of the individuals in the affected populations. (2) Offering targeted treatment to a group within a community where the group may be defined by age, gender, religion or other social characteristics, such as primary school children. (3) Offering selective treatment based on intensity of current or past infection.

A series of studies in Nigerian villages compared the efficacy of different treatment strategies, namely universal, targeted and selective treatment with levamisole (Asaolu, Holland \& Crompton, 1991). Both universal and targeted treatment of schoolchildren were identified as the most effective approaches to control both in terms of reducing intensity in a high risk group and having an effect on intensity in untreated adults. Bundy et al. (1990) obtained similar results in a study of children infected with both $T$. trichiura and $A$. lumbricoides (aged 2-15 years) on the island of Montserrat, in which the prevalence and intensity of both infections declined not only in the treated group, but also in the 16-25 year old group that received no treatment.

In contrast, a study of 880 people in Dhaka, Bangladesh treated with pyrantel three times at 6 month intervals (Hall, Anwar \& Tomkins, 1992), revealed that nearly two-thirds of all subjects were heavily infected at least once over the 18 month period and that universal chemotherapy was a more appropriate control strategy with which to reduce the level of Ascaris infection in the community. The authors concluded that if treatment at rounds 2 and 3 had been provided only to those people who were heavily infected at round 1, 155 subjects would not have been treated, and that over the duration of the study these 155 subjects would have become heavily re-infected at least once. 


\section{Chemotherapy regimes}

Several re-infection studies of a population group treated for Ascaris have indicated that re-infection rates reach the pre-control level within 6 to 12 months (Tu et al. 1972; Cabrera, Arambulo \& Portillo, 1975 ; Arfaa \& Ghadirian, 1977; Thazin Oo, 1977; Croll et al. 1982; Seo, 1983; Yodmani et al. 1983 ; Cabrera, 1984). Thein Hlaing et al. (1987) showed that 6-monthly chemotherapy reduced intensity in both children and adults but that 12monthly treatment lowered intensity only in adults. In a later study Thein Hlaing et al. (1991 b) observed that 3-monthly chemotherapy with levamisole gave better results in terms of both prevalence and intensity than 4-monthly and 6-monthly treatments. For the 3 chemotherapeutic regimes used in the study and provided at 3-monthly intervals targeted at children under 15 years old, (a) with and (b) without universal chemotherapy, and (c) at 2-12 years old children over a period of 2 years, all were almost equally effective in reducing the prevalence and intensity not only in target children but also in non-target adults. Similar findings were reported for 4-monthly (Cabrera \& Cruz, 1983; Holland et al. 1996a) and 3-monthly (Cabrera et al. 1989) chemotherapeutic treatment regimes in children under 15 years of age. Other studies also reported reductions in the prevalence and intensity of ascariasis in untreated adults within communities where children have been targeted for treatment (Thein Hlaing et al. 1990, 1991; Bundy et al. 1990).

\section{Choice of anthelminthic drug in control programmes}

According to Albonico et al. (1999) the choice of anthelminthic drug in public health should be tailored to the local epidemiology of soil-transmitted helminth infections. This is why information should be collected on prevalence and intensity of helminth infections, the population groups at highest risk of morbidity, and the health impact of helminth infections in the community that can benefit from treatment. Furthermore, the choice of anthelminthic drug for use in a control programme depends on (1) its safety record, (2) its therapeutic effect (cure rate or efficacy), (3) its spectrum of activity, (4) local health policy, and (5) financial considerations. A key issue for the optimal use of an anthelminthic drug is to decide when and how frequently to treat the population of concern. The results of epidemiological surveys to determine re-infection rates are also useful for determining the treatment schedules (Hall et al. 1992).

A number of studies have compared the efficacy of various anthelminthic drugs in communities polyparasitised by soil-transmitted helminths. Hadju et al. (1996b) compared the efficacy of pyrantel and albendazole in 6-11 year old children in South Sulawesi, Indonesia who were infected with both $A$. lumbricoides and $T$. trichiura. Five hundred and seven children were assigned randomly to 4 anthelminthic groups (pyrantel or albendazole once or twice per year) and a placebo, according to gender and A. lumbricoides egg counts. Children with signs of severe protein malnutrition were excluded. Children received single doses of pyrantel, albendazole or placebo at baseline, 3, 6 and 12 months. During the 12-month follow-up however, only 330 children $(65 \%)$ completed all exams. No important differences were noted at baseline between those children and those who completed the study. In addition, no differences were found between the five groups at baseline in prevalence and intensity of both infections. At the end of the study, both albendazole and pyrantel groups had significantly lower prevalences of $A$. lumbricoides than the placebo group $(P<0 \cdot 05)$. At the 12 month examination, the intensity of ascariasis in the twice dosed albendazole and pyrantel groups was significantly lower than the placebo group $(P<0.005$ and $P<0.0005$, respectively). The results indicated that two doses yearly of each drug were more effective than one dose yearly.

Many studies have reported highly effective single dose treatments for $A$. lumbricoides. For example Sinniah, Chew \& Subramaniam (1990), Albonico et al. (1994) and Rahman (1996) have all observed that both albendazole and mebendazole can be very effective in eliminating Ascaris. Williams, Koroma \& Hodges (1997) found that both levamisole and albendazole significantly reduced both the prevalence and intensity of Ascaris. Marti et al. (1996) found both ivermectin and albendazole to be just as effective. In deciding the anthelminthic of choice in the treatment of ascariasis, health planners need to consider not just the cost of available drugs but also their efficacy against other human soil-transmitted helminths such as hookworm, T. trichiura, and Strongyloides stercoralis. Infections of A. lumbricoides are rarely found alone in human communities (Crompton, 1994). Many of the above studies have reported mixed results in the efficacies of the drugs against these other helminth species. For example, as albendazole is effective against both the adult and ova/larval stages of hookworm, unlike pyrantel, it is therefore necessary to retreat at-risk populations more frequently with pyrantel than with albendazole (Williams et al. 1997). Similarly, single-dose albendazole treatment was reported to be more effective than mebendazole against hookworm (Holzer \& Frey, 1987; Ismail, Premaratne \& Suraweera, 1991 ; Albonico et al. 1994; Rahman, 1996). For this reason administration of more expensive drugs cannot be justified in communities in which parasitic infections besides Ascaris are not prevalent or are of low prevalence. 
D ISCUSSION

Many studies have investigated the contribution of ascariasis to child malnutrition in spite of the difficulties in imputing causality posed by polyparasitism (Keusch \& Migasena, 1982; Watkins \& Pollitt, 1996). When polyparasitised hosts are dewormed with broad-spectrum drugs, it is difficult to determine the extent to which the improvements seen are due to each parasite species treated. This is one reason why further studies should be undertaken to differentiate between the pathogenic effects of individual intestinal helminth species on their hosts.

The potential mechanisms responsible for reduced growth and other developmental insults of Ascaris infection (described in detail by Stephenson \& Holland, 1987; Taren \& Crompton, 1989) function primarily via reductions in nutrient intake, and to a lesser extent, decreases in digestion, absorption and nutrient utilization (see Fig. 3 and Anorexia in Malnutrition and Parasitic Helminth Infections, this volume). However, because the relationship between ascariasis and child malnutrition is conditional and is one that depends on the interaction of multiple biomedical and behavioural factors (Cerf, Rohde \& Soesanto, 1981), not every study undertaken to examine this relationship is successful in demonstrating that anthelminthic treatment can lead to improved growth. Stephenson \& Holland (1987), Thein Hlaing (1993) and Watkins et al. (1996) have outlined other reasons why some studies have not demonstrated improved growth in children treated for Ascaris infection; these include: (1) Sub-optimal use of chemotherapeutic drug; e.g. see Greenberg $e t$ al. (1981); (2) Combination of factors including the absence of severe malnutrition in the population, relatively adequate dietary energy and protein intakes, possible low worm burdens and/or a failure to eliminate ascariasis, e.g. see Gupta \& Urrutia, 1982; Kightlinger et al. 1996; (3) Presence of other parasites such as Giardia lamblia in a study area against which anthelminthic drugs such as mebendazole or piperazine are ineffective; e.g. see Gupta \& Urrutia (1982); Thein Hlaing (1993); (4) Presence of other adverse environmental influences and infectious diseases, including diarrhoea and acute respiratory infections; e.g. see Ismail \& Perara (1986); (5) Small sample size and insufficient time for follow-up examination, e.g. see Freij et al. (1979); (6) Communities where Ascaris prevalence is low and where there are different worm populations; e.g. see Watkins et al. 1996. The authors suggested that one reason why only a modest gain in weight was observed in Guatemalan children compared with the four Kenyan studies conducted within the same population (Adams et al. 1994; Stephenson et al. $1993 a, b, 1989)$ was that hookworm infections strongly influenced the latter studies. However the populations also differed in that the Guatemalan children were stunted, showing past chronic malnutrition but little current acute malnutrition, and the Kenyan children exhibited both forms of malnutrition.

Connolly \& Kvalsvig(1993)outlinetwobroadclasses of causal connection by which parasitic infection may impair cognitive function. The first involves direct action of the parasitic agent itself on the hosts central nervous system and the impact this may have on behaviour or mental processes. The second is that by causing ill-health and debilitation a parasite can indirectly restrict the activities of the host to the extent that certain intellectual skills are less readily acquired compared to others who are not affected in this way. Connolly \& Kvalsvig (1993) propose the hypothesis that a general effect of parasitic infection is to limit the energy resources available to infected individuals and adversely affect motivation, emotion and patterns of social interaction, and that these in turn will affect their capacity for physical and mental work in a variety of contexts (e.g. school, home, play, work). This hypothesis clearly requires further investigation. The question of whether or not the effects are irreversible where there is gross insult to the central nervous system also needs further investigation.

According to Thein Hlaing et al. (1991 b), there are two possible explanations for the substantial reductions in worm intensity that occur with agetargeted community chemotherapy. Firstly, children in the community harbour most of the total adult worm population and therefore pass most of the eggs that infect others (Thein Hlaing et al. 1984; Elkins, Haswell-Elkins \& Anderson, 1986). Secondly, regular and repeated treatments heighten the impact of chemotherapy because of the predisposition to acquire heavy infection (in children) or light infections (in adults) of Ascaris (Haswell-Elkins, Elkins \& Anderson, 1987; Thein Hlaing et al. 1987).

Since A. lumbricoides infections usually co-exist with other intestinal infections, compelling reasons exist for promoting control programmes aimed at soil-transmitted helminthiases generally. A. lumbricoides is readily expelled in response to a single oral dose of anthelminthic drugs (Crompton, 1994); this makes the results of chemotherapy an ideal focus for health education lessons about improved personal hygiene and sanitation. Since the control of such infections in developing countries requires the deployment of scarce resources, the optimal choice of treatment strategy must be based on analysis of the financial costs of each type of intervention as well as on the public health significance (Holland et al. 1996b). Albonico et al. (1995) highlighted the importance of data on prevalence and intensity for planning, monitoring and evaluating large-scale helminth control programmes, so that each control strategy can be tailored to the local epidemiological situation. 
Results of a study in four Nigerian villages to compare the effects of universal, targeted and selective chemotherapy (Asaolu et al. 1991) showed that both universal and targeted treatments were the most effective regimes on the basis of a reduction of 1000 eggs per $\mathrm{g}$ of faeces (Holland et al. 1996b). For the number treated, the selective approach was 17 times more expensive than universal treatment. Targeted treatment was only twice the cost per person treated relative to the universal approach. The targeted approach carries other significant advantages, including the case of reaching children through attendance at school (Stephenson et al. $1980 \mathrm{~b}$ ), the reduced possibility of disrupting acquired immunity in the adult classes (Keymer \& Pagal, 1990), the avoidance of concern over teratogenic effects (Bundy \& Cooper, 1989) and in the way schools can greatly facilitate the administration of anthelminthic treatment as a centre for drug delivery where modern health education can also be developed and integrated with the chemotherapy (Crompton, 1994). However, because school enrolment and attendance rates can be low in some developing countries, significant numbers of children will not receive anthelminthic treatment in a targeted programme (Albonico et al. 1999).

In contrast, the selective approach would seem in theory to be a particularly attractive option since the use of relatively few doses of drugs would reduce costs and perhaps help delay or avoid conditions that might lead to the development of drug resistance (Crompton, 1994). In practice, however, the disadvantages of this type of treatment are that heavily infected people must be identified before they are treated and that not only are most people likely to be infected at least once over a relatively short period of repeated treatment, but a large proportion are likely to be heavily infected (Hall et al. 1992). Also, selectivity may cause resentment, particularly in endemic communities where the need for treatment is a general perception (Holland et al. 1996b).

The economic evaluation of control programmes in developing countries is important in part because it introduces concepts of cost analysis and gives an indication of affordability. Guyatt, Bundy \& Evans (1993) undertook a cost effectiveness examination of different frequencies of universal chemotherapy using cost data from an actual control programme for A. lumbricoides (Bundy et al. 1990). Using a dynamic model of helminth infection transmission (Medley, Guyatt \& Bundy, 1993), the analysis suggested that for a strategy with defined control objectives, budgetary constraints and a specific rate of transmission, it could be more cost-effective to intervene in a high transmission area than in a low transmission area. Also, the analysis showed that employing relatively long intervals between treatments offers the best results. Even though child-targeted treatment can never be more effective than treatment of the total population, Guyatt et al. (1995) found, in a follow-up analysis of the same data source, that because children tend to have higher intensities of infection, child-targeted treatment can be more costeffective than population treatment in reducing the number of disease cases. Furthermore, the same analysis suggested that expanding the proportion of a population covered could be a more cost-effective approach then increasing frequency of treatment.

\section{REFERENCES}

ADAMS, E. J., STEPHENSON, L. S., LATHAM, M. C. \& KinOTi, S. N. (1994). Physical activity and growth of Kenyan school children with hookworm, Trichuris trichiura and Ascaris lumbricoides infections are improved after treatment with albendazole. Fournal of Nutrition 124, 1199-1206.

akamatsu, т. (1959). Study of the experimental infection with Ascaris in babies and infants. Nippon Shonika Gakkai Zasshi 62, 1584-1592.

Albonico, M., CROMpton, D. W. T. \& SAVioli, L. (1999). Control strategies for human intestinal nematode infections. Advances in Parasitology 42, 278-341.

albonico, M., Smith, P. G., hall, A., ChWaya, H. M., ALAWI, K. S. \& SAVIOLI, L. (1994). A randomized controlled trial comparing mebendazole and albendazole against Ascaris, Trichuris and hookworm infections. Transactions of the Royal Society of Tropical Medicine and Hygiene 88, 585-589.

ALbONico, M., SMith, P. G., ERCOle, E., Hall, A., ChWAYA, H. M., ALAWI, K. S. \& SAVIOLI, L. (1995). Rate of reinfection with intestinal nematodes after treatment of children with mebendazole or albendazole in a highly endemic area. Transactions of the Royal Society of Tropical Medicine and Hygiene 89, $538-541$.

Anderson, R. M. (1989). Transmission dynamics of Ascaris lumbricoides and the impact of chemotherapy. In Ascariasis and its Prevention and Control (ed. Crompton, D. W. T., Nesheim, M. C. \& Pawlowski, Z. S.), pp. 253-273. London and Philadelphia, Taylor and Francis.

ANDERSON, R. M. \& MAY, R. M. (1991). Infectious Diseases of Humans. Dynamics and Control. Oxford, Oxford University Press.

Anderson, T. J. C. (1995). Ascaris infections in humans from North America: molecular evidence for crossinfection. Parasitology 110, 215-219.

ANDERSON, T. J. C., ROMERO-ABAL, M. E. \& JAENiKe, J. (1993). Genetic structure and epidemiology of Ascaris populations: patterns of host affiliation in Guatemala. Parasitology 107, 319-334.

ARFAa, F. \& GHADIRIAN, E. (1977). Epidemiology and mass treatment of ascariasis in six rural communities in Central Iran. American Fournal of Tropical Medicine and Hygiene 26, 866-871.

ASAOLU, s. O., HOLland, C. v. \& CROMPTON, D. w. T. (1991). Community control of Ascaris lumbricoides in rural Oyo State, Nigeria: Mass, targeted and selective treatment with levamisole. Parasitology 103, 291-298. 
ASAOLU, S. O., HOLland, C. V., JEGEDE, J. O., FRASER, N. R., STODDARD, R. C. \& CROMPTON, D. W. T. (1992). The prevalence and intensity of soil-transmitted helminthiases in rural communities in Southern Nigeria. Annals of Tropical Medicine and Parasitology 86, 279-287.

BEAver, P. C. \& DANARAJ, T. J. (1958). Pulmonary ascariasis resembling eosinophilic lung: autopsy report with description of larvae in the bronchioles. American Fournal of Tropical Medicine and Hygiene 7 , 100-111.

BEAVER, P. C., JUNG, R. C. \& CUPP, E. W. (1984). Oxyuroidea and Ascaridoidea. In Clinical Parasitology. 9th edn. Philadelphia, Lea and Febiger.

BEISEL, W. R. ( 1982). Synergism and antagonism of parasitic diseases and malnutrition. Reviews of Infectious Diseases 4, 746-750.

Blumenthal, D. S. \& schultz, M. G. (1976). Effects of Ascaris infection on nutritional status in children. American Fournal of Tropical Medicine and Hygiene 25, 682-690.

BIDINGER, P. D., CROMPTON, D. W. T. \& ARNOLD, S. (1981). Aspects of intestinal parasitism in villages from rural peninsular India. Parasitology 83, 373-380.

BUNDY, D. A. P. \& COOPER, E. S. (1989). Trichuris and trichuriasis in humans. Advances in Parasitology 28, 107-173.

BUNDY, D. A. P., WONG, M. S., LEWIS, L. L. \& HORTON, J. (1990). Control of geohelminths by delivery of targeted chemotherapy through schools. Transactions of the Royal Society of Tropical Medicine and Hygiene 84, 115-120.

CABRERA, B. D. (1984). Reinfection and infection rates of ascariasis in relation to seasonal variation in the Philippines. Southeast Asian Fournal of Tropical Medicine and Public Health 15, 394-401.

CABrera, B. D., arambulo, P. v. \& Portillo, G. P. (1975). Ascariasis control and/or eradication in a rural community in the Philippines. Southeast Asian Fournal of Tropical Medicine and Public Health 6, 510-518.

CABrera, B. D., CABAllero, B., RAMPAL, L. \& DE LEON, W. (1989). National experiences of Ascariasis control in Philippines. In Ascariasis and its Prevention and Control (ed. Crompton, D. W. T., Nesheim, M. C. \& Pawlowski, Z. S.), pp. 169-183. London and Philadelphia, Taylor and Francis.

CABrera, B. D. \& CRUZ, A. C. (1983). A comparative study on the effect of mass treatment in the entire community and selective treatment of children on the total prevalence of soil-transmitted helminthiases in two communities, Mindoro, Philippines. In Collected Papers on the Control of Soil-Transmitted Helminthiases, Vol. II (ed. Yokogawa, M., Hayashi, S., Kobayashi, A., Kagei, N., Suzuki, N. \& Kunii, C.), pp. 266-287. Tokyo, Asian Parasite Control Organization.

CABRERA, B. D. \& VALEZA, F. (1980). The reinfection rate of soil-transmitted helminths in the pilot areas after treatment. In Collected Papers on the Control of SoilTransmitted Helminthiases, Vol. I (ed. Yokogawa, M., Hayashi, S., Kobayashi, A., Kagei, N., Suzuki, N. \& Kunii, C.), pp. 141-149. Tokyo, Asian Parasite Control Organization.
CARRERA, E., NeSheim, M. C. \& CROMPton, D. W. T. (1984). Lactose maldigestion in Ascaris-infected preschool children. American Fournal of Clinical Nutrition 39, 255-264.

CERF, B. J., Rohde, J. E. \& SOesanto, T. (1981). Ascaris and malnutrition in a Balinese village: a conditional relationship. Tropical and Geographical Medicine 33, 367-373.

CHAI, J. Y., CHO, S. Y., LEE, S. H. \& SEO, B. S. (1991). Reduction in the incidence of biliary and other surgical complications of ascariasis according to the decrease of its national egg prevalence in Korea. Korean Fournal of Parasitology 29, 101-111.

CHAN, M. S., Medley, G. F., JAMison, D. \& BUNDY, D. A. P. (1994). The evaluation of potential global mortality attributable to intestinal nematode infections. Parasitology 109, 373-387.

CHANDiWANA, s. K., BRAdley, M. \& CHOMBo, F. (1989). Hookworm and roundworm infections in farm-worker communities in the large-scale agricultural sector in Zimbabwe. Fournal of Tropical Medicine and Hygiene 92, 338-344.

CHRUNGOO, R. K., HANGloo, V. K., FAROQUi, M. M. \& KHAN, M. (1992). Surgical manifestations and management of ascariasis in Kashmir. Fournal of the Indian Medical Association 90, 171-174.

COLES, G. C. (1985). Allergy and immunopathology of ascariasis. In Ascariasis and its Public Health Significance (ed. Crompton, D. W. T., Nesheim, M. C. \& Pawlowski, Z. S.), pp. 167-184. London and Philadelphia, Taylor and Francis.

CONNOLly, K. J. \& KVAlsvig, J. D. (1993). Infection, nutrition and cognitive performance in children. Parasitology 107, S187-S200.

CROLl, N. A., ANDERSON, R. M., GYORKOS, T. W. \& GHADIRIAN, E. (1982). The population biology of Ascaris lumbricoides in a rural community in Iran. Transactions of the Royal Society of Tropical Medicine and Hygiene 76, 187-97.

CROLL, N. A. \& GHADIRIAN, F. (1981). Wormy persons: Contributions to the nature and patterns of overdispersion with Ascaris lumbricoides, Ancyclostoma duodenale, Necator americanus and Trichuris trichiura. Tropical and Geographical Medicine 33, 241-248.

CROMPTON, D. W. T. (1992). Ascariasis and childhood malnutrition. Transactions of the Royal Society of Tropical Medicine and Hygiene 86, 577-579.

Crompton, D. w. T. (1994). Ascaris lumbricoides. In Parasitic and Infectious Diseases (ed. Scott, M. E. and Smith, G.) chapter 14, pp. 175-196. London and New York, Academic Press Inc.

CROMPTON, D. W. T. \& SAVIOLI, L. (1993). Intestinal parasitic infections and urbanization. Bulletin of the World Health Organization 71, 1-7.

CROMPton, D. W. T. \& Tulley, J. J. (1987). How much ascariasis is there in Africa? Parasitology Today 3, $123-127$

Cross, J. H., Clarke, M. D., DURFEe, P. T., IRVING, G. S., TAYlor, J., PARTONO, F., JOESOEF, A., Hudojo, \& oemijati, s. (1975). Parasitology survey and seroepidemiology of amoebiasis in South Kalimantan (Borneo), Indonesia. Southeast Asian Fournal of Tropical Medicine and Public Health 6, 52-60. 
CURTALE, F., VAIDYA, Y., MUHILAL, \& TILDEN, R. L. (1994). Ascariasis, hookworm infection and serum retinol amongst children in Nepal. Panminerva Medicina 36, 19-21.

DE SILVA, N. R., CHAN, M. S. \& BUNDY, D. A. P. (1997 $a$ ). Morbidity and mortality due to ascariasis : reestimation and sensitivity analysis of global numbers at risk. Tropical Medicine and International Health 2, 519-528.

DE SILVA, N. R., GUYATT, H. L. \& BUNDY, P. (1997b). Morbidity and mortality due to Ascaris-induced intestinal obstruction. Transactions of the Royal Society of Tropical Medicine and Hygiene 91, 31-36.

EL-ARABY, I. L., EL-DiN, M. K. B. \& ABDOU, M. O. (1984). A study on cell-mediated immunity in children with ascariasis. Saudi Medical Fournal 5, 37-40.

ELKINS, D. B., HASWELl-ELKINS, M. \& ANDERSON, R. M. (1986). The epidemiology and control of intestinal helminths in the Pulicat Lake region of Southern India. 1. Study design and pre- and post-treatment observations on Ascaris lumbricoides infection. Transactions of the Royal Society of Tropical Medicine and Hygiene 80, 774-792.

ESREy, S. A., Potash, J. B., Roberts, L. \& SHiff, C. (1991). Effects of improved water supplies and sanitation on ascariasis, diarrhoea, dracunculiasis, hookworm infection, schistosomiasis, and trachoma. Bulletin of the World Health Organization 69, 609-621.

FORREster, J. E. \& SCOTT, M. E. (1990). Measurement of Ascaris lumbricoides infection intensity and the dynamics of expulsion following treatment with mebendazole. Parasitology 100, 303-308.

FORRESTER, J. E., SCOTT, M. E., BUNDY, D. A. P. \& GOLDEN, M. H. N. (1988). Clustering of Ascaris lumbricoides and Trichuris trichiura infections within households. Transactions of the Royal Society of Tropical Medicine and Hygiene 82, 282-288.

FREIJ, L., MEEUWISSE, G. W., BERG, N. O., WALl, S. \& GEBRE-MEDHIN, M. (1979). Ascariasis and malnutrition. A study in urban Ethiopian children. American Fournal of Clinical Nutrition 32, 1545-1553.

FURNEE, C. A., WEST, C. E., VAN DER HAAR, F. \& HAUTVAST, J. G. A. J. (1997). Effect of intestinal parasite treatment on the efficacy of oral iodized oil for correcting iodine deficiency in schoolchildren. American Fournal of Clinical Nutrition 66, 1422-1427.

GALVIN, T. J. (1968). Development of human and pig Ascaris in the pig and rabbit. Fournal of Parasitology 54, 1085-1091.

GElPhi, A. P. \& MUSTAFA, A. (1967). Seasonal pneumonitis with eosinophilia: A study of larval ascariasis in Saudi Arabs. American Fournal of Tropical Medicine and Hygiene 16, 646-657.

GILlES, H. M. (1985). Selective primary health care: strategies for control of disease in the developing world. XVII. Hookworm infection and anaemia. Reviews of Infectious Diseases 7, 111-118.

GREENBERG, B. L., GILMAN, R. H., SHAPIRO, H., GILMAN, J. B., MONDAL, G., MAKSUd, M., Khatoon, H. \& CHOWDHuRy, J. (1981). Single dose piperazine therapy for Ascaris lumbricoides: an unsuccessful method of promoting growth. American Fournal of Clinical Nutrition 34, 2508-2516.
GROVE, D. A. (1990). Ascaris lumbricoides and ascariasis. In A History of Human Helminthology, (ed. Grove, D. A.), pp. 469-497. Wallingford, UK, C. A. B. International.

GUPTA, M. C. (1985). Ascariasis and malnutrition in children: studies in India and Guatemala. In Ascariasis and its Public Health Significance (ed. Crompton, D. W. T., Nesheim, M. C. \& Pawlowski, Z. S.), pp. 203-211. London and Philadelphia, Taylor and Francis.

GUPTA, M. C., Mithal, S., ARORA, K. L. \& TANDON, B. N. (1977). Effect of periodic deworming on nutritional status of Ascaris infected pre-school children receiving supplementary food. The Lancet 2, 108-10.

GUPTA, M. C. \& URRUTia, J. J. (1982). Effect of periodic antiascaris and antigiardia treatment on nutritional status of pre-school children. American Fournal of Clinical Nutrition 36, 79-86.

GUYATT, H. L., BUNDY, D. A. P. \& EVANS, D. (1993). A population dynamic approach to the cost-effectiveness analysis of mass anthelmithic treatment: effects of treatment frequency on Ascaris infection. Transactions of the Royal Society of Tropical Medicine and Hygiene 87, 570-575.

GUyatt, H. L., Chan, M. S., Medley, G. F. \& BUNDy, D. A. P. (1995). Control of Ascaris infection by chemotherapy: which is the most cost-effective option? Transactions of the Royal Society of Tropical Medicine and Hygiene 89, 16-20.

HADIDJAJA, P., BONANG, E., SUYARDI, M. A., ABIDIN, S. A., ISMID, I. S. \& MARGANO, s. S. (1998). The effect of intervention on nutritional status and cognitive function of primary school children infected with Ascaris lumbricoides. American Fournal of Tropical Medicine and Hygiene 59, 791-795.

hadju, V., STEPHENSON, L. S., ABADi, K., MOHAMmed, H. O., BOWMAN, D. D. \& PARKER, R. S. (1996a). Improvement in appetite and growth in helminthinfected schoolboys three and seven weeks after a single dose of pyrantel pamoate. Parasitology 113, 497-504.

HADJU, V., STEPHENSON, L., SATRIONO, BOWMAN, D., MOHAMmed, H. \& ABADI, K. (1996b). Comparison between albendazole and pyrantel pamoate once and twice yearly in urban slum school children in Ujung Pandang. Medical Fournal of Indonesia 5, 195-202.

HADJU, V., STEPHENSON, L. S., MOHAMMED, H. O., BOWMAN, D. D. \& PARKER, R. S. (1998). Improvements of growth, appetite, and physical activity in helminthinfected schoolboys six months after a single dose of albendazole. Asia Pacific Fournal of Clinical Nutrition 7, 170-176.

HALL, A. (1982). Intestinal helminths of man: the interpretation of egg counts. Parasitology 85, 605-613.

HALl, A., ANWAR, K. S. \& TOMKINS, A. M. (1992). Intensity of reinfection with Ascaris lumbricoides and its implications for parasite control. The Lancet 339, 1253-1257.

HALl, A., ANWAR, K. S., TOMKINS, A. M. \& RAHMAN, L. (1999). The distribution of Ascaris lumbricoides in human hosts: a study of 1765 people in Bangladesh. Transactions of the Royal Society of Tropical Medicine and Hygiene 93, 1-8. 
HARINASUTA, T. \& CHAROENLARP, P. (1980). The nematode infections in Thailand. In Collected Papers on the Control of Soil-transmitted Helminthiases. (ed. Yokogawa, M., Hayashi, S., Kobayashi, A., Kagei, N., Suzuki, N. \& Kunii, C.), Vol. I, pp. 87-93. Tokyo, Asian Parasite Control Organization.

HASWELL-ELKINS, M. R., ELKINS, D. \& ANDERSON, R. M. (1989). The influence of individual, social group and household factors on the distribution of Ascaris lumbricoides within a community and implications for control strategies. Parasitology 98, 125-134.

HASWELl-Elkins, M., ElKins, D. B. \& ANDERSON, R. M. (1987). Evidence for predisposition in humans to reinfection with Ascaris, hookworm, Enterobius and Trichuris in a southern Indian fishing community. Parasitology 95, 323-337.

HENRY, F. J. (1988). Reinfection with Ascaris lumbricoides after chemotherapy: a comparative study in three villages with varying sanitation. Transactions of the Royal Society of Tropical Medicine and Hygiene 82, 460-464.

ново, в. (1956). Epidemiological studies on Ascaris infection among prisoners and the length of life of Ascaris lumbricoides in human host. Fapanese Fournal of the Nation's Health 25, 1-14.

holland, C. V., ASAOlu, s. O., CROMPton, D. W. T., STODDARD, R. C., MCDONALD, R. \& TORIMIRO, S. E. A. (1989). The epidemiology of Ascaris lumbricoides and other soil-transmitted helminths in primary school children from Ile-Ife, Nigeria. Parasitology 99, 275-285.

holland, C. V., ASAOLU, s. o., CROMPton, D. W. T., Whitehead, R. R. \& COOMbs, I. (1996a). Targeted anthelminthic treatment of schoolchildren: effect of frequency of application on the intensity of Ascaris lumbricoides infection in children from rural Nigerian villages. Parasitology 113, 87-95.

HOLLAND, C. V., CROMPTON, D. W. T., ASAOLU, s. O., CRICHTON, W. B., TORIMiro, S. E. A. \& WALters, D. E. (1992). A possible genetic factor influencing protection from infection with Ascaris lumbricoides in Nigerian children. Fournal of Parasitology 78 , 915-916.

holland, C. v., o'shea, E., asaolu, S. o., TURLEy, o. \& CRompton, D. W. T. (1996b). A cost-effectiveness analysis of anthelminthic intervention for community control of soil-transmitted helminth infection: levamisole and Ascaris lumbricoides. Fournal of Parasitology 82, 527-530.

HOLland, C. V., TAREN, D. L., CROMPTON, D. W. T., NESHEIM, M. C., SANJUR, D., BARBEAU, I., TUCKER, K., TIFFANY, J. \& RIVERA, G. (1988). Intestinal

helminthiases in relation to the socioeconomic environment of Panamanian children. Social Science and Medicine 26, 209-213.

HOLZER, B. R. \& FREY, F. J. (1987). Differential efficacy of mebendazole and albendazole against Necator americanus but not for Trichuris trichiura infestations. European Fournal of Clinical Pharmacology 32, 635-637.

HUtTLY, S. R. A. (1990). The impact of inadequate sanitary conditions on health in developing countries. World Health Statistics Quarterly 43, 118-126.
ISMAIL, M. M. \& PERERA, W. D. A. (1986). Relationship between soil-transmitted helminthiases and nutritional status in 3-12-year olds in semi-urban community in Sri Lanka. In Collected Papers on the Control of Soiltransmitted Helminthiases (ed. Yokogawa, M., Hayashi, S., Kobayashi, A., Kagei, N., Suzuki, N. \& Kunii, C.), Vol. III, pp. 186-199. Tokyo, Asian Parasite Control Organization.

ismail, M. M., PRemaratne, U. N. \& SURaweera, M. G. (1991). Comparative efficacy of single dose anthelminthics in relation to intensity of geohelminths infections. Ceylon Medical Fournal 36, 162-167.

JARretT, E. E. \& Miller, H. R. (1982). Production and activities of $\mathrm{IgE}$ in helminth infection. In Immunity and Concomitant Immunity in Infectious Diseases (Kallos, P. ed.). Progress in Allergy 31, 178-233. KAGEI, N. (1983). Techniques for the measurement of environmental pollution by infective stage of soiltransmitted helminths. In Collected Papers on the Control of Soil-transmitted Helminthiases (ed. Yokogawa, M., Hayashi, S., Kobayashi, A., Kagei, N., Suzuki, N. \& Kunii, C.), Vol. II pp. 27-46. Tokyo, Asian Parasite Control Organization.

KAN, s. P., GUYATT, H. L. \& BUNDY, D. A. P. (1989). Geohelminth infection of children from rural plantations and urban slums in Malaysia. Transactions of the Royal Society of Tropical Medicine and Hygiene 83, 817-820.

KeusCh, G. T. \& Migasena, P. (1982). Biological implications of polyparasitism. Review of Infectious Disease 4, 880-882.

KEYMER, A. E. (1982). Density-dependent mechanisms in the regulation of intestinal helminth populations. Parasitology 84, 573-587.

KeYMeR, A. E. \& PAGAL, M. (1990). Predisposition to helminth infection. In Hookworm Disease : Current Status and New Directions (ed. Schad, G. A. \& Warren, K. S.), pp. 177-209. London and Philadelphia, Taylor and Francis.

KHUROO, M. S., ZARGAR, S. A. \& MAHAJAN, R. (1990). Hepatobiliary and pancreatic ascariasis in India. The Lancet (June 23) 1503-1506.

KIGHTLINGER, L. K., SEED, J. R. \& KIGHTLINGER, M. B. (1996). Ascaris lumbricoides aggregation in relation to child growth status, delayed cutaneous hypersensitivity, and plant anthelminthic use in Madagascar. Fournal of Parasitology 82, 25-33.

KIGHTLINGER, L. K., SEED, J. R. \& KIGHTLINGER, M. B. (1998). Ascaris lumbricoides intensity in relation to environmental, socioeconomic, and behavioural determinants of exposure to infection in children from Southeast Madagascar. Fournal of Parasitology 84, 480-484.

KRasnonos, L. N. (1978). Prolonged survival of Ascaris lumbricoides L., 1758 ova in the soil in Samarkand. Meditsinskata Parazitolila I Paraziarnye Bolezni 47, 103-105. (Cited in Helminthological Abstracts 1979, 411).

Kroeger, A., SChUlz, B., Witte, B., SKeWES-RAMM, R. \& ETZLER, A. (1992). Helminthiases and cultural change in the Peruvian rainforest. Fournal of Tropical Medicine and Hygiene 95, 104-113.

KVAlsvig, J. D., COOPPAN, R. M. \& CONNOlly, K. J. (1991). 
The effects of parasitic infections on cognitive process in children. Annals of Tropical Medicine and Parasitology 85, 551-568.

LYNCH, N. R., HAGEL, I., PEREZ, M., DI PRISCO, M., AlVAREZ, N. \& ROJAS, E. (1992b). Bronchoconstriction in helminthic infection. International Archives of Allergy and Immunology 98, 77-79.

LYNCH, N. R., ISTURIZ, G., SANCHEZ, Y., PEREZ, M., MARTINEZ, A. \& CASTES, M. (1992a). Bronchial challenge of tropical asthmatics with Ascaris lumbricoides. Fournal of Investigational Allergology and Clinical Immunology 2, 97-105.

LYNCH, N. R., PALENQUe, M., HAGEL, I. \& DIPRISCO, M. C. (1997). Clinical improvement of asthma after anthelminthic treatment in a tropical situation. American Journal of Respiratory and Critical Care Medicine 156, 50-54.

LYSEK, H. (1967). On the host specificity of ascarids of human and pig origin. Helminthologia 8, 309-312.

MaChado, M. T., MACHADO, T. M. S., YoshikaE, R. M., SCHMIDT, A. L. A., FARIA, R. A., PASCHOALOTTI, M. A., BARATA, R. B. \& CHIEFFI, P. P. (1996). Ascariasis in the subdistrict of Cavacos, Municipality of Alterosa (MG), Brazil: Effect of mass treatment with albendazole on the intensity of infection. Revista do Instituto do Medicina Tropical de Sao Paulo 38, 265-271.

MaRTi, H., Haji, H. J., SAVioli, L., ChWAYa, H. M., MGENi, A. F., AMEIR, J. S. \& HATZ, C. (1996). A comparative trial of a single-dose ivermectin versus three days of albendazole for treatment of Strongyloides stercoralis and other soil-transmitted helminth infections in children. American Fournal of Tropical Medicine and Hygiene 55, 477-481.

Mccullough, F. (1974). Observations on of Ascaris lumbricoides infection in Mwanza, Tanzania. In Parasites in Man and Animals in Africa (ed. Anderson, C. \& Kilama, W. L.), pp. 359-385. Nairobi, East African Literature Bureau.

MCSHARRY, C., XIA, Y., HOLLAND, C. v. \& KENNEDY, M. W. (1999). Natural Immunity to Ascaris lumbricoides associated with immunoglobulin $\mathrm{E}$ antibody to ABA-1 allergen and inflammation indicators in children. Infection and Immunity 67, 1-6.

MEDLEy, G. F., GUYATT, H. L. \& BUNDY, D. A. P. (1993). A quantitative framework for evaluating the effects of community treatment on the morbidity due to ascariasis. Parasitology 106, 211-221.

MURREll, K. D., ERIKSEN, L., NANSEN, P., SLOTVED, H.-C. \& Rasmussen, т. (1997). Ascaris suum: A revision of its early migratory path and implications for human ascariasis. Fournal of Parasitology 83, 255-260.

Nichols, R. L. (1956). The etiology of visceral larva migrans. II. Comparative larval morphology of Ascaris lumbricoides, Necator americanus, Strongyloides stercoralis and Ancylostoma caninum. Fournal of Parasitology 42, 363-399.

PAWlowski, Z. s. (1978). Ascariasis. Clinics in Gastroenterology 7, 157-178.

PAWLOWSKI, Z. S. \& ARFAA, F. (1984). Ascariasis. In Tropical and Geographical Medicine (ed. Warren, K. S. \& Mahmoud, A. A. F.), pp. 347-358. New York, McGraw Hill.
PAWLOWSKi, Z. S. \& DAVIS, A. (1989). Morbidity and mortality in ascariasis. In Ascariasis and its Prevention and Control (ed. Crompton, D. W. T., Nesheim, M. C. \& Pawlowski, Z. S.), pp. 71-86. London and Philadelphia, Taylor and Francis.

PENG, W., ANDERSON, T. J. C., ZHOU, X. \& KENNEDY, M. W. (1998). Genetic variation in sympatric Ascaris populations from humans and pigs in China. Parasitology 117, 355-361.

PENG, W., ZHOU, X., CUI, X., CROMPTON, D. W. T., WHiteHEAD, R. R., XIONG, J., WU, H., PENG, J., YANG, Y., WU, W., XU, K. \& YAN, Y. (1996). Ascaris, people and pigs in a rural community of Jiangxi Province, China. Parasitology 113, 545-557.

PETERS, w. (1978). Medical aspects-comments and discussion II. Symposia of the British Society for Parasitology 16, 25-40. Oxford, Blackwell Scientific Press.

PINUS, J. (1985). Surgical complications of ascariasis in Brazil. In Ascariasis and its Public Health Significance (ed. Crompton, D. W. T., Nesheim, M. C. \& Pawlowski, Z. S.), pp. 161-166. London and Philadelphia, Taylor and Francis.

PRost, A. (1987). L'ascaridiose en Afrique de l'ouset. Revue épidémiologique. Annales de Parasitologie Humaine et Comparée 62, 434-455.

PURI, S. \& CHANDRA, R. K. (1985). Nutritional regulation of host resistance and predictive value of immunologic tests in assessment of outcome. Pediatric Clinics of North America 32, 499-516.

RAHMAN, W. A. (1996). Comparative trials using albendazole and mebendazole in the treatment of soiltransmitted helminths in schoolchildren on Penang, Malaysia. Southeast Asian Fournal of Tropical Medicine and Public Health 27, 765-767.

SAVIOLI, L., BUNDY, D. \& TOMKINS, A. (1992). Intestinal parasitic infections: a soluble public health problem. Transactions of the Royal Society Medicine and Hygiene 86, 353-354.

SCRIMShaw, N. S. \& SANGIOvanNi, J. P. (1997). Synergism of nutrition, infection, and immunity: an overview. American Fournal of Clinical Nutrition 66, 464S-477S. SEO, B. S. (1983). Control problems of ascariasis in Korea with special reference on the related biology and epidemiology. In Collected Papers on the Control of Soil-Transmitted Helminthiases, Vol. II (ed. Yokogawa, M., Hayashi, S., Kobayashi, A., Kagei, N., Suzuki, N. \& Kunii, C.), pp. 194-216. Tokyo, Asian Parasite Control Organization.

SHIELD, J. M., SCRIMGEOUR, E. M. \& VATERLAWS, A. L. (1980). Intestinal helminths in an adult hospital population in the Eastern Highlands of Papua New Guinea: Relationship with anemia, eosinophilia and asthma. Papua New Guinea Medical Fournal 23, 157-164.

SinNiah, B. (1982). Daily egg production of Ascaris lumbricoides: The distribution of eggs in the faeces and the variability of egg counts. Parasitology $\mathbf{8 4}$, 167-175.

SinNiah, B., CHEW, P. I. \& SUbramaniam, K. (1990). A comparative trial of albendazole, mebendazole, pyrantel pamoate and oxantel pyrantel pamoate against soil transmitted helminthiases in school children. Tropical Biomedicine 7, 129-134. 
STEPHENSON, L. S., CROMPTON, D. W. T., LATHAM, M. C., SCHUlpen, J., NESHEIM, M. C. \& JANSEN, A. A. J. (1980a). Relationships between Ascaris infection and growth of malnourished preschool children in Kenya. American Fournal of Clinical Nutrition 33, 1165-1172.

STEPHenson, L. S. \& HOLland, C. v. (1987). The Impact of Helminth Infections on Human Nutrition. London and Philadelphia, Taylor and Francis.

STEPHENSON, L. S., LATHAM, M. C., ADAMS, E. J., KinOti, S. N. \& PERTET, A. (1993 $a$ ). Weight gain of Kenyan school children infected with hookworm, Trichuris trichiura, and Ascaris lumbricoides is improved following once- or twice-yearly treatment with albendazole. Fournal of Nutrition 123, 656-665.

STEPHENSON, L. S., LATHAM, M. C., ADAMS, E. J., Kinoti, S. N. \& PERTET, A. $(1993 b)$. Physical fitness, growth and appetite of Kenyan school boys with hookworm, Trichuris trichiura and Ascaris lumbricoides are improved four months after a single dose of albendazole. Fournal of Nutrition 123, 1036-1046.

STEPHENSON, L. S., LATHAM, M. C., KURZ, K. M., KinOTi, s. N. \& BRINGHAM, H. (1989). Treatment with a single dose of albendazole improves growth of Kenyan schoolchildren with hookworm, Trichuris trichiura, and Ascaris lumbricoides infections. American Fournal of Tropical Medicine and Hygiene 41, 78-87.

STEPHENSON, L. S., LATHAM, M. C. \& ODUORI, M. L. $(1980 \mathrm{~b})$. Costs, prevalence and approaches to control of ascariasis in Kenya. Fournal of Tropical Pediatrics 26, 246-264.

STOREY, G. W. \& PHillips, R. A. (1985). The survival of parasite eggs throughout the soil profile. Parasitology 91, 585-590.

TAKata, I. (1951). Experimental infection of man with Ascaris of man and the pig. Kitasato Archives of Experimental Medicine 23, 49-59.

TANUMiHardjo, S. A., PERMAESIH, D., MUHERDIYANTININGSIH, RUSTAN, E., RUSMIL, K., FATAH, A. C., Wilbur, S., MUhilal, Kariyadi, D. \& Olson, J. A. (1996). Vitamin A status of Indonesian children infected with Ascaris lumbricoides after dosing with vitamin A supplements and albendazole. Fournal of Nutrition 126, 451-457.

TAREN, D. L. \& CROMPTON, D. W. T. (1989). Nutrition interactions during parasitism. Clinical Nutrition $\mathbf{8}$, 227-238.

TAREN, D. L., NESHEIM, M. C., CROMPTON, D. W. T., HOLland, C. V., BARBEAU, I., RIVERA, G., SANJUR, D., TIFFANY, J. \& TUCKER, K. (1987). Contributions of ascariasis to poor nutritional status in children from Chiriqui Province, Republic of Panama. Parasitology 95, 603-613.

THAZIN Oo (1977) Reinfection rate of ascariasis and evaluation of mass chemotherapy for control of ascariasis. M.Sc. (Zoology), Rangoon Arts and Science University.

THEIN HLAING (1993). Ascariasis and childhood malnutrition. Parasitology 107, S125-S136.

THEIN HLAING, MYAT LAY KYIN, HLAING MYA \& MAUNG MAUNG (1990). Role of ascariasis in surgical abdominal emergencies in the Rangoon Children's Hospital Burma. Annals of Tropical Paediatrics 10, 53-60.

THEIN HLAING, THAN SAW \& MYAT LAY KYIN (1991 b). The impact of three-monthly age-targeted chemotherapy on Ascaris lumbricoides infection. Transactions of the Royal Society of Tropical Medicine and Hygiene $\mathbf{8 1}$, 519-522.

THEIN HLAING, THAN SAW, HTAY HTAY AYE, MYINT LWIN \& THEIN MAUNG MYINT (1984). Epidemiology and transmission dynamics of Ascaris lumbricoides in Okpo village, rural Burma. Transactions of the Royal Society of Tropical Medicine and Hygiene 78, 497-504. THEIN HLAING, THAN SAW \& MYINT LWIN (1987). Reinfection of people with Ascaris lumbricoides following single, 6-months and 12-month interval mass chemotherapy in Okpo village, rural Burma. Transactions of the Royal Society of Tropical Medicine and Hygiene 81, 140-146.

THEIN HLAING, THANE TOE, THAN SAW, MYAT LAY KYIN \& MYINT LWIN (1991 a). A controlled chemotherapeutic intervention trial on the relationship between Ascaris lumbricoides infection and malnutrition in children. Transactions of the Royal Society of Tropical Medicine and Hygiene 85, 523-528.

THIENPONT, D., ROCHETTE, F. \& VANPARIJS, O. F. J. (1986). Diagnosing Helminthiases by Coprological Examination. 2nd edn. Beerse, Belgium, Janssen Research Foundation.

Tomkins, A. \& WATSOn, F. (1989). Malnutrition and Infection. Geneva, World Health Organization.

TRIPATHY, K., DUQUe, E., Bolanos, o., LOTERO, H. \& MAYORAL, L. G. (1972). Malabsorption syndrome in ascariasis. American Fournal of Clinical Nutrition 25, 1276-1287.

TU, M., KHIN OHN LWIN, THAW SAW \& HTAY AUNG (1972). Reinfection with Ascaris lumbricoides after anthelminthic therapy. Abstracts of the Seventh Burma Research Congress, pp. 134-135. Rangoon.

voGEL, H. \& MINNING, w. (1942). Beitrage zur klinik der lungen-ascariasis und zur frage der flüchtigen eosinophilen lungeninfiltrate. Beiträge zur Klinic der Tuberkulose 98, 624-654.

WALsh, J. A. \& WARREN, K. s. (1979). Selective primary health care. New England Fournal of Medicine 301, 967-974.

Watkins, W. E., CRuz, J. R. \& POllitt, E. (1996). The effects of deworming on indicators of school performance in Guatemala. Transactions of the Royal Society of Tropical Medicine and Hygiene 90, 156-161.

WATKins, W. E. \& POLlitT, E. (1996). Effects of removing Ascaris on the growth of Guatemalan schoolchildren. Pediatrics 97, 871-876.

Willett, W. C., Kilama, W. L. \& Kihamia, C. M. (1979). Ascaris and growth rates: a randomized trial of treatment. American Fournal of Public Health 69, 987-991.

Williams, R. A. M., Koroma, M. M. \& HodGes, M. (1997). Comparison of albendazole and levamisole chemotherapy on prevalence and intensity of common soil-transmitted helminth infections in school children, Sierra Leone. West African Fournal of Medicine 16, 179-183.

WILliAMS-BLANGERO, S., SUBEDI, J., UPADHAYAY, R. P., MANRAL, D. B., RAI, D. R., JHA, B., ROBINSON, E. S. \& BLANGERO, J. (1999). Genetic analysis of susceptibility to infection with Ascaris lumbricoides. American Fournal of Tropical Medicine \& Hygiene 60, 921-926. WONG, M. S. \& BUNDY, D. A. P. (1990). Quantitative 
assessment of contamination of soil by the eggs of Ascaris lumbricoides and Trichuris trichiura.

Transactions of the Royal Society of Tropical Medicine and Hygiene 84, 567-570.

WONG, M. S., BUNDY, D. A. P. \& GOLDEN, M. H. N. (1988). Quantitative assessment of geophageous behaviour as a potential source of exposure to geohelminth infection. Transactions of the Royal Society of Tropical Medicine and Hygiene 82, 621-625.

WONG, M. S., BUNDY, D. A. P. \& GOLDEN, M. H. N. (1991).

The rate of ingestion of Ascaris lumbricoides and Trichuris trichiura eggs in soil and its relationship to infection in two children's homes in Jamaica.

Transactions of the Royal Society of Tropical Medicine and Hygiene 85, 89-91.

WORLD health organization (1967). Control of Ascariasis. Report of a World Health Organization expert committee. WHO Technical Report Series No. 379. Geneva, World Health Organization.

WORLD HEALTH ORGANIZATION (1981). Intestinal Protozoan and Helminthic Infections. Report of a World Health Organization scientific group. WHO Technical Report Series No. 666. Geneva, World Health Organization.

WORLd health ORganization (1985a). Diagnostic Techniques for Intestinal Parasitic Infections (IPI) Applicable to Primary Health Care (PHC) Services. LIHO/P.D.P./85.2 Geneva, World Health Organization.

WORLD HEALTH ORGanization (1985b). General
Strategies for Prevention and Control of Intestinal Parasitic Infections (IPI) within Primary Health Care (PHC). LIHO/P.D.P./85'1. Geneva, World Health Organization.

WORld health organization (1987). Prevention and Control of Intestinal Parasitic Infections. WHO Technical Report Series No. 749. Geneva, World Health Organization.

world health organization (1992). Bench Aids for the Diagnosis of Intestinal Helminths. WHO/CDS/IPI. Geneva, World Health Organization.

WORLD HEALTH ORGanization (1996a). The World Health Report 1996 : Fighting Disease. Fostering Development. Geneva, World Health Organization. WORLD HEALTH ORGANIZATION (1996b). Report of the WHO Informal Consultation on Hookworm and Anaemia in Girls and Women. Division of Control of Tropical Diseases. WHO/CTD/SIP.96•1. Geneva, World Health Organization.

YODMANI, B., SORNMANI, S., PHATIHATAKORN, W. \& HARINASUTA, C. (1983). Reinfection of ascariasis after treatment with pyrantel pamoate and the factors relating to its active transmission in a slum in Bangkok. In Collected Papers on the Control of SoilTransmitted Helminthiases, Vol. II (ed. Yokogawa, M., Hayashi, S., Kobayashi, A., Kagei, N., Suzuki, N. \& Kunii, C.), pp. 89-100. Tokyo, Asian Parasite Control Organization.

yoshida, s. (1919). On the development of Ascaris lumbricoides L. Fournal of Parasitology 5, 105-115. 